\title{
EchoGéo
}

$28 \mid 2014$

Police : les espaces de l'ordre, l'ordre en espace

\section{Le Cap en noir? La Mother City dans les romans policiers de Deon Meyer}

\section{Martine Berger}

\section{(2) OpenEdition}

1 Journals

Édition électronique

URL : https://journals.openedition.org/echogeo/13863

DOI : $10.4000 /$ echogeo. 13863

ISSN : 1963-1197

\section{Éditeur}

Pôle de recherche pour l'organisation et la diffusion de l'information géographique (CNRS UMR 8586)

Référence électronique

Martine Berger, « Le Cap en noir ? La Mother City dans les romans policiers de Deon Meyer », EchoGéo [En ligne], 28 | 2014, mis en ligne le 08 juillet 2014, consulté le 31 juillet 2021. URL : http:// journals.openedition.org/echogeo/13863; DOI : https://doi.org/10.4000/echogeo.13863

Ce document a été généré automatiquement le 31 juillet 2021.

EchoGéo est mis à disposition selon les termes de la licence Creative Commons Attribution - Pas d'Utilisation Commerciale - Pas de Modification 4.0 International (CC BY-NC-ND) 


\title{
Le Cap en noir? La Mother City dans les romans policiers de Deon Meyer
}

\author{
Martine Berger
}

\section{NOTE DE L'ÉDITEUR}

Les coordinatrices de ce numéro ont souhaité permettre une lecture à 2 niveaux de cet article très riche et documenté sur Deon Meyer. Au lecteur familier de cet auteur, la possibilité d'affiner ses connaissances en parcourant l'article dans son intégralité y compris les nombreuses références et citations en italique dans le texte ; au lecteur plus pressé ou moins au fait des ouvrages de Déon Meyer, la possibilité de contourner ces paragraphes sans pour autant perdre la qualité et le fil de l'article.

1 Comme l'ont souligné de nombreux auteurs' ${ }^{1}$, le roman noir se veut témoin de l'état du monde et de la société : pour Jean-Patrick Manchette, par exemple, « le grand polar est son époque ». Sa dimension critique en fait l'une des manières de rendre compte des bouleversements de la société. Pour Caryl Ferey, auteur français tenté par le dépaysement dans l'Afrique du Sud post-apartheid ${ }^{2}$, « il se trouve que nous n'imaginons pas le monde sans contexte politique et social, voire ethnique, que nous refusons la position de l'autruche [..], préférant nous coltiner le réel, même amoché $»^{3}$. Donner du sens au réel, décrypter les fractures d'une société et de ses territoires, lire dans les dysfonctionnements du présent - la forte incidence du crime, la récurrence de la violence et de la corruption, l'injustice sociale au quotidien - les traces d'un lourd passé mais peut être aussi les germes d'un avenir meilleur, tel est aussi le projet de nombreux écrivains sud-africains. Plusieurs d'entre eux, dont deux sont d'anciens journalistes ${ }^{4}$, ont choisi de le faire à travers la forme du roman policier, dans des œuvres qui se déroulent, au moins pour partie, au Cap.

Dans la littérature policière, la ville constitue souvent un personnage à part entière, et ce n'est sans doute pas un hasard si Le Cap, plus encore que Johannesburg, où les actes de violence sont souvent plus spectaculaires, impose sa présence. Berceau de la 
littérature de contestation de l'apartheid, de l'ouverture au monde au temps des sanctions et du boycott international, Le Cap est aussi un port, avec ses entrepôts désaffectés, porte d'entrée pour des mafias étrangères qui «modernisent » le crime en important de nouveaux objets et moyens de délit, en profitant de l'inscription de la ville dans la toile de la mondialisation.

Des trois auteurs sud-africains ${ }^{5}$ qui prennent Le Cap comme théâtre principal de leurs romans noirs, Deon Meyer est sans doute aujourd'hui celui qui rend le mieux compte de la ville, dans sa diversité, ses contradictions, ses mouvements. Non seulement par l'ampleur de son œuvre ${ }^{6}$ qui comporte des personnages récurrents dont la biographie s'enrichit au fil des romans, mais aussi parce que ses ouvrages sont largement ouverts sur la société civile, le quotidien des habitants « ordinaires » d'une grande métropole, la manière dont ils se logent et se déplacent, se rencontrent ou s'évitent. Ce souci de précision documentaire apparaît bien dans les postfaces de ses romans, où il remercie ses informateurs dans différents milieux professionnels, et cite de nombreuses références bibliographiques et les sites web qu'il a consultés. Dans À la trace (Tracks, 2010), l'éditeur français a même pris soin d'insérer des cartes permettant de suivre au plus près les déplacements des personnages. Une raison de plus, pour des géographes, de s'intéresser à ce que Deon Meyer nous dit de l'espace capetonien et de sa société, de leur place dans l'Afrique du Sud post-apartheid.

\section{Une géographie sociale de l'espace capetonien}

4 Les romans de Deon Meyer ${ }^{7}$, tout particulièrement les trois derniers (Thirteen Hours, 2010 ; Trackers, 2011, et Seven Days, 2012), fourmillent de détails permettant de localiser où habitent et travaillent les personnages principaux et secondaires, le cadre de leurs rencontres, de leurs achats et de leurs loisirs, ainsi que l'environnement urbain des crimes et délits, invitant le lecteur à un jeu de piste dans les quartiers du Cap ${ }^{8}$, ou à défaut sur un plan'.

\section{Dis-moi où tu habites, je te dirai qui tu es}

5 Il est rare que les quartiers, voire les immeubles, où résident les personnages ne soient pas localisés (quartier, rue, parfois numéro) et décrits avec précision, dans le souci d'une grande fidélité au style urbanistique et à la diversité sociale des lieux. On voyage ainsi des banlieues nord à dominante afrikaner, où résident nombre de personnages appartenant aux classes moyennes blanches, aux espaces les plus sélectifs du piémont de la Montagne de la Table, à Constantia (la propriété d'Henry van Eeden, intermédiaire financier dans des transactions relevant du $\mathrm{BEE}^{10}$, dans Trackers), des lotissements anciens aux gated communities les plus récentes. Ou encore des quartiers résidentiels centraux d'appartements ou de villas sur les pentes (Green Point et Sea Point, Oranjezicht et Tamboerskloof) au premier cercle des banlieues anciennes, qui logeaient autrefois des employés et ouvriers blancs, et sont aujourd'hui en cours de gentrification résidentielle ou commerciale, avec la rénovation du tissu pavillonnaire et la reconquête d'anciens ateliers ou entrepôts (Woodstock, Observatory) ${ }^{11}$. 
Illustration 1 - Dans les quartiers résidentiels du centre : Oranjezicht

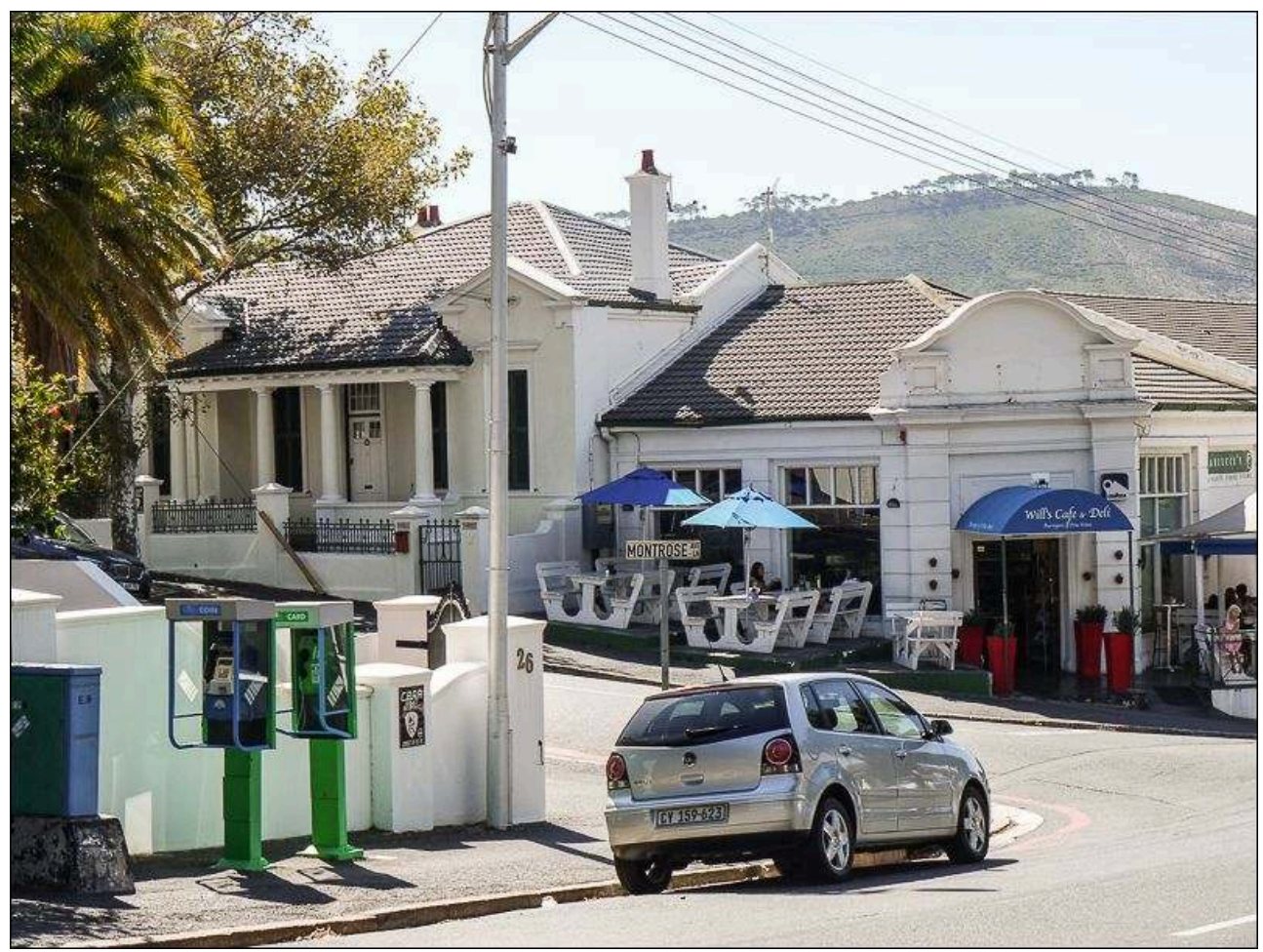

Le café Carlucci et une villa victorienne, comme celle habitée par le professeur (Thirteen Hours). Auteur: M. Houssay-Holzschuch, avril 2014.

6 Autre quartier résidentiel du centre, Tamboerskloof, « était censée être une banlieue chic » (Thirteen Hours). 
Illustration 2 - Tamboerskloof, Brownlow Road

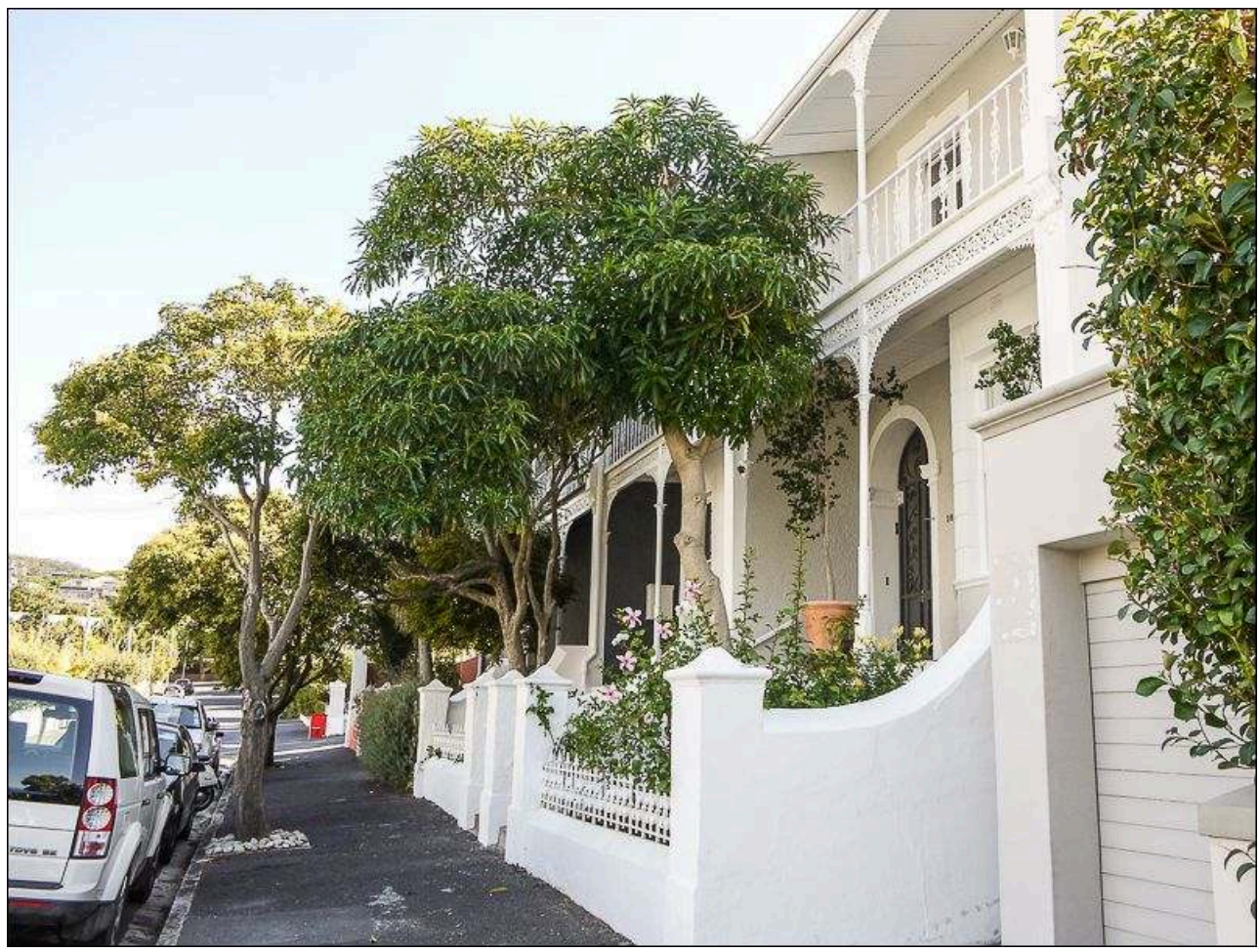

Brownlow Road et ses vieilles maisons victoriennes : « La $n^{\circ} 47$ était immense et impressionnante avec ses deux étages, ses vérandas et ses balcons aux balustrades en fer forgé, ses murs crème et ses fenêtres aux volets verts » (là où habite Alexa Barnard, ex-star de la pop music afrikaner).

Auteur : M. Berger, avril 2014 
Illustration 3 - Tamboerskloof, Higgo Road

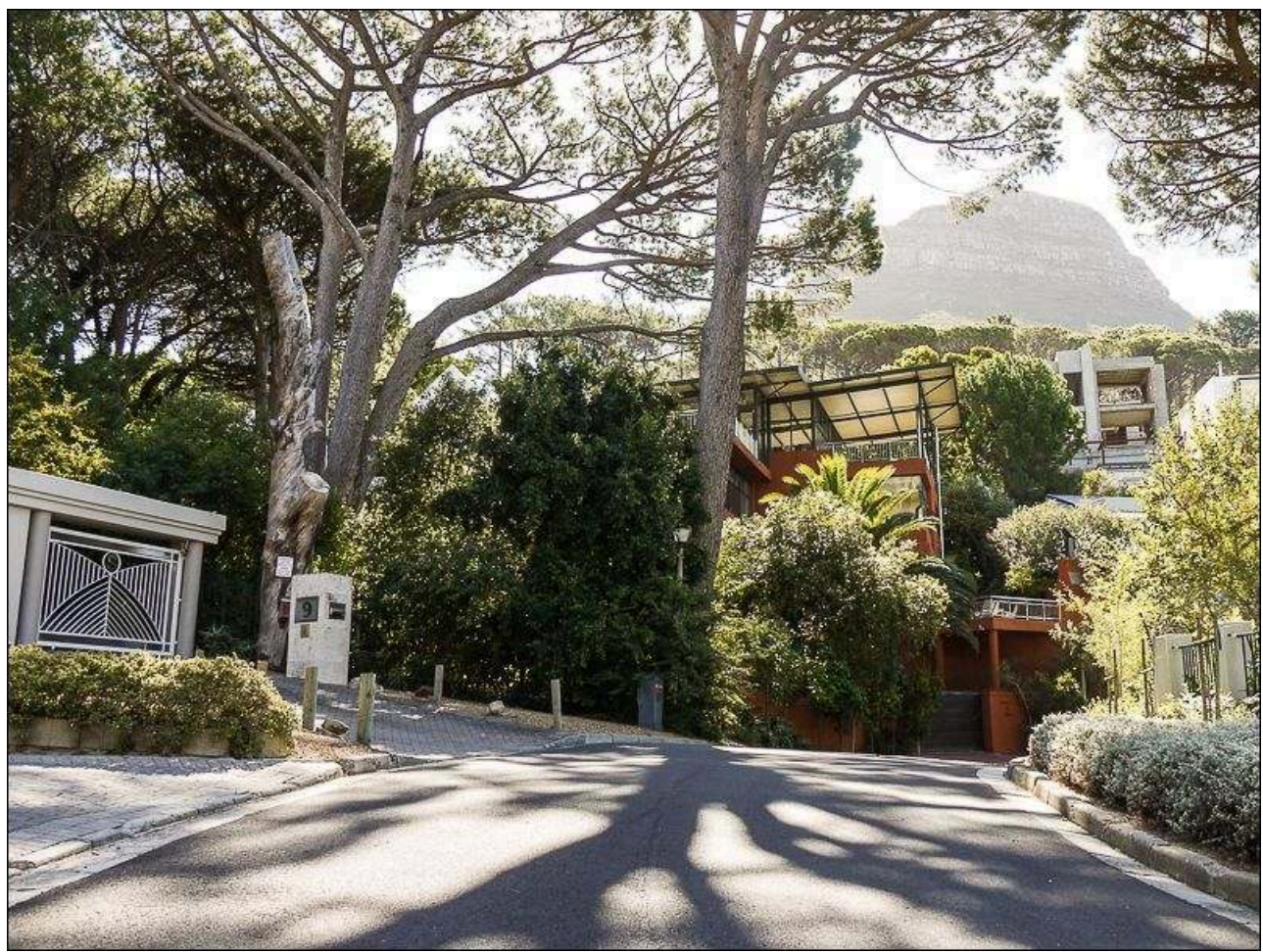

Higgo Road : «Immenses et luxueuses demeures entourées de jardins à végétation luxuriante et protégés par de hauts murs ». "Toutes les grilles étaient cadenassées, chaque maison était un véritable fortin ».

Auteur: M. Houssay-Holzschuch, avril 2014.

$7 \quad$ Mais il s'agit pour l'essentiel de la ville blanche : si les Noirs et les Coloureds sont très présents sur les lieux du travail, souvent à des postes de responsabilité, leurs quartiers de résidence sont plus rarement décrits. Les townships, curieusement, apparaissent peu dans les romans de Deon Meyer : parfois (rarement) comme les lieux des crimes - dans Devil's Peak, les violeurs et auteurs de crimes rituels contre de jeunes enfants opèrent principalement à Khayelitsha, Mitchell's Plain, Bonteheuwel, Bishop Lavis, Valhalla Park, Manenberg ou Crossroads - ou comme des réservoirs de jeunes désœuvrés pour les gangs (Manenberg); plus rarement comme des lieux où habitent, pourtant, une majorité des Capetoniens. Leur immensité, leur uniformité, semblent défier tout repère, d'autant que si, dans la réalité, ils sont effectivement dotés de noms de rues improbables ${ }^{12}$, il est rare que des plaques permettent aux personnes étrangères au quartier d'y trouver leur chemin. 
Illustration 4a - Township : Khayelitsha

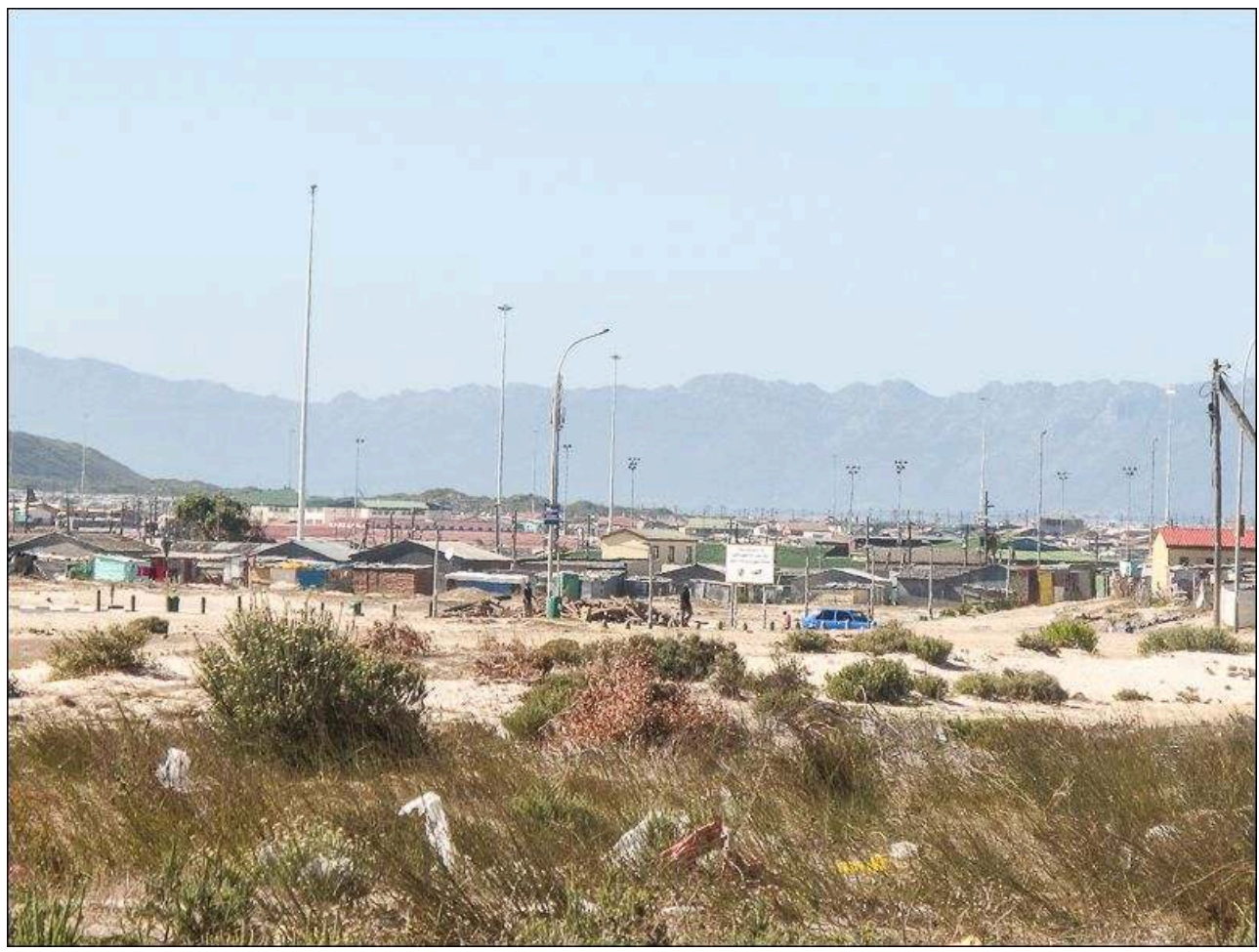

Khayelitsha : « Une ville à l'écart de la ville, s'étirant à travers des dunes sablonneuses », où « il était facile de se perdre » (Devil's Peak).

Auteur: M. Berger, avril 2011

Illustration 4b - Township : Khayelitsha

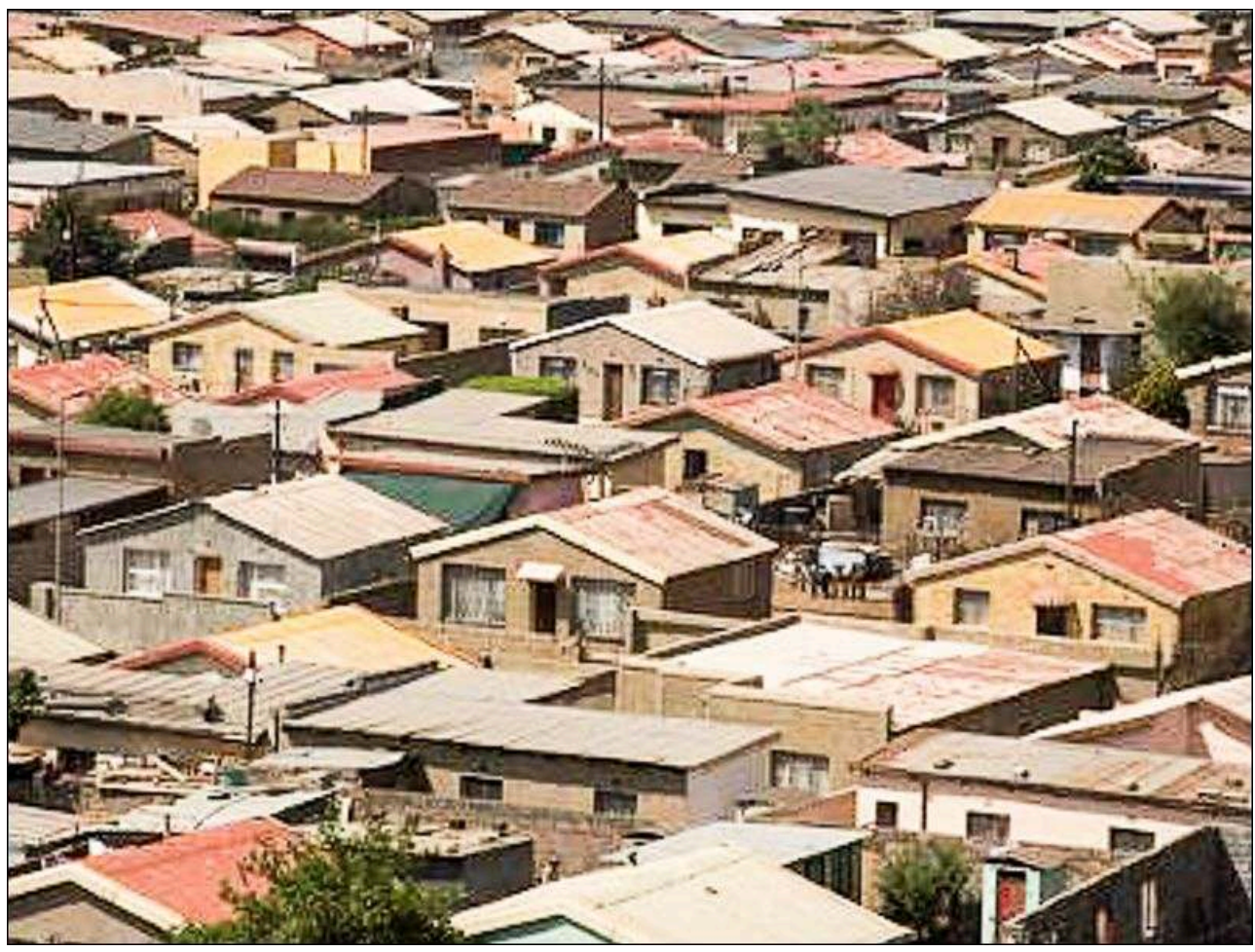

Auteur: J. Koshi, 2008. 


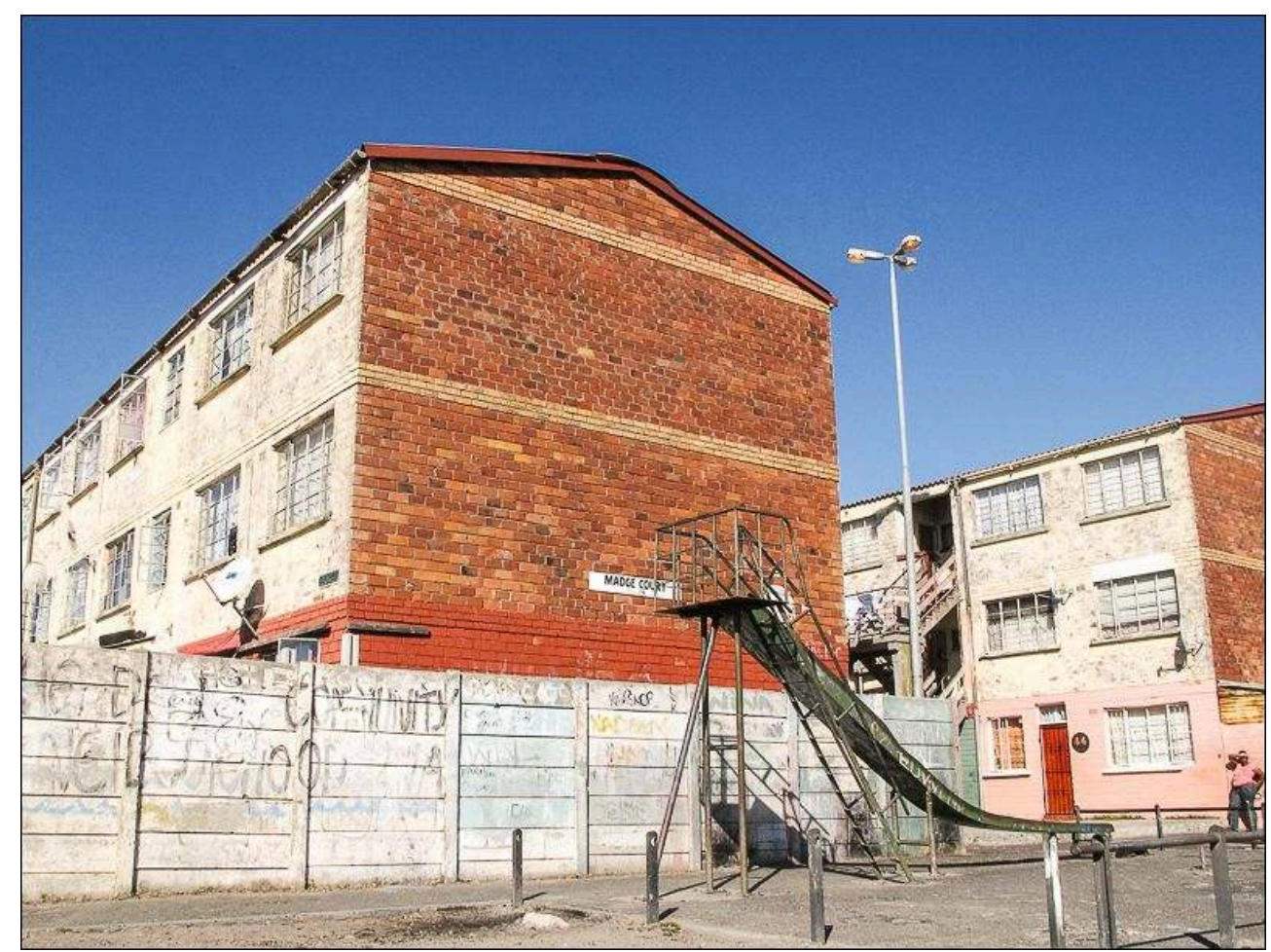

Manenberg : Où le gang des Restless Ravens recrute ses hommes de main (Trackers).

Auteur : M. Berger, avril 2012.

Ainsi, Khayelitsha est décrite par Thobela Mpayipheli - grand Noir xhosa qui fut, dans une vie antérieure, soldat dans la Lutte et exécutant des basses œuvres de la Stasi, aujourd'hui rangé et homme à tout faire chez un concessionnaire de motos BMW dans le City Bowl - comme « une ville à l'écart de la ville, s'étirant à travers des dunes sablonneuses", où "il était facile de se perdre", faute de poteaux indicateurs (Devil's Peak). Il habite un moment à Gugulethu où sa compagne, Miriam Nzululwazi, a acheté, avec ses économies et un emprunt contracté auprès d'une banque, une petite maison et où elle entretient avec Thobela un petit jardin potager : un meilleur environnement pour élever son fils, et plus proche de son travail à l'agence ABSA d'Heerengracht (dans le City Bowl), que Khayelitsha où elle habitait précédemment (Heart of the Hunter, 2003). Mais après le décès de Miriam, Thobela, qui a acheté une ferme dans les collines du Transkei, près de l'endroit où il est né, part avec son fils vers des horizons plus calmes. Vusumuzi Ndabeni, jeune policier xhosa qui vient d'être promu et affecté à une nouvelle unité, la Brigade d'intervention de la province, a été 4 ans en poste à Khayelitsha, "qui lui avait souvent brisé le cœur avec ses meurtres gratuits, ses violences domestiques, sa terrible pauvreté, ses taudis, son combat de chaque jour. Mais il avait été le bienvenu là-bas, celui qui faisait régner la loi et l'ordre. Les gens simples, les gens du peuple, le respectaient, l'aidaient, le soutenaient » (Thirteen Hours, p. 201).

Il est rare que Deon Meyer indique une adresse précise dans les townships, qui sont identifiés par des noms de quartiers, décrits comme des "êtres collectifs", aux alignements de maisons monotones, matchboxes ou nouvelles constructions dans le cadre du RDP ${ }^{13}$. Font exception la maison de Miriam, Govan Mbeki Road, sur une des artères bordant Gugulethu, township ancien, en cours d'amélioration, voire de gentrification (Teppo et Houssay-Holzschuch, 2013). Et, concernant les townships coloureds des Cape Flats, la référence au quartier de Sabie Street Courts, décrit comme 
l'un des plus durs de Manenberg, d'où vient K.D. Snyders, garde du corps de Terry (Terror) Richard Baadjies, le "général» des Restless Ravens. Si uniformes et indifférenciés qu'ils puissent paraître au premier coup d'œil, les townships n'en sont pas moins un assemblage de quartiers diversifiés, où les hauts-lieux du crime et des gangs ne constituent que des points isolés sur la carte des Cape Flats, où vit une population respectable, et laborieuse, quand elle le peut.

9 Tout aussi diverses que les Cape Flats, les banlieues Nord juxtaposent des quartiers de standing différent, marqueurs de la réussite sociale, ou du déclassement, de leurs habitants. Les policiers, fussent-ils inspecteurs, ne peuvent prétendre habiter les meilleurs quartiers, là où se commettent souvent les crimes, en dépit des multiples dispositifs de sécurité. On y côtoie plus souvent des chefs d'entreprise, et c'est aussi là que s'abritent les têtes dirigeantes du crime organisé.

Il faut des circonstances exceptionnelles (une ambition démesurée et un sens politique aigu pour Janina Mentz, directrice-adjointe puis directrice de l'Agence Présidentielle de Renseignement; une épouse ayant une belle situation dans une grande entreprise, pour Fransman Dekker, inspecteur de police coloured, ambitieux lui aussi) pour accéder à ces quartiers de rêve (Bantry Bay, Clifton, ou Camps Bay sur le littoral atlantique sud, Tygerberg ou Milnerton Ridge au Nord) lorsqu'on est salarié de l'État ou de la Province.

«L'argent méritait de gagner car la vue était d'une beauté stupéfiante. Voilà à quoi servait l'argent. À réserver le plus beau pour les riches. Et à expédier les flics à Brackenfell »(Devil's Peak, p. 381).

Illustration 6a - Les banlieues Nord : Durbanville

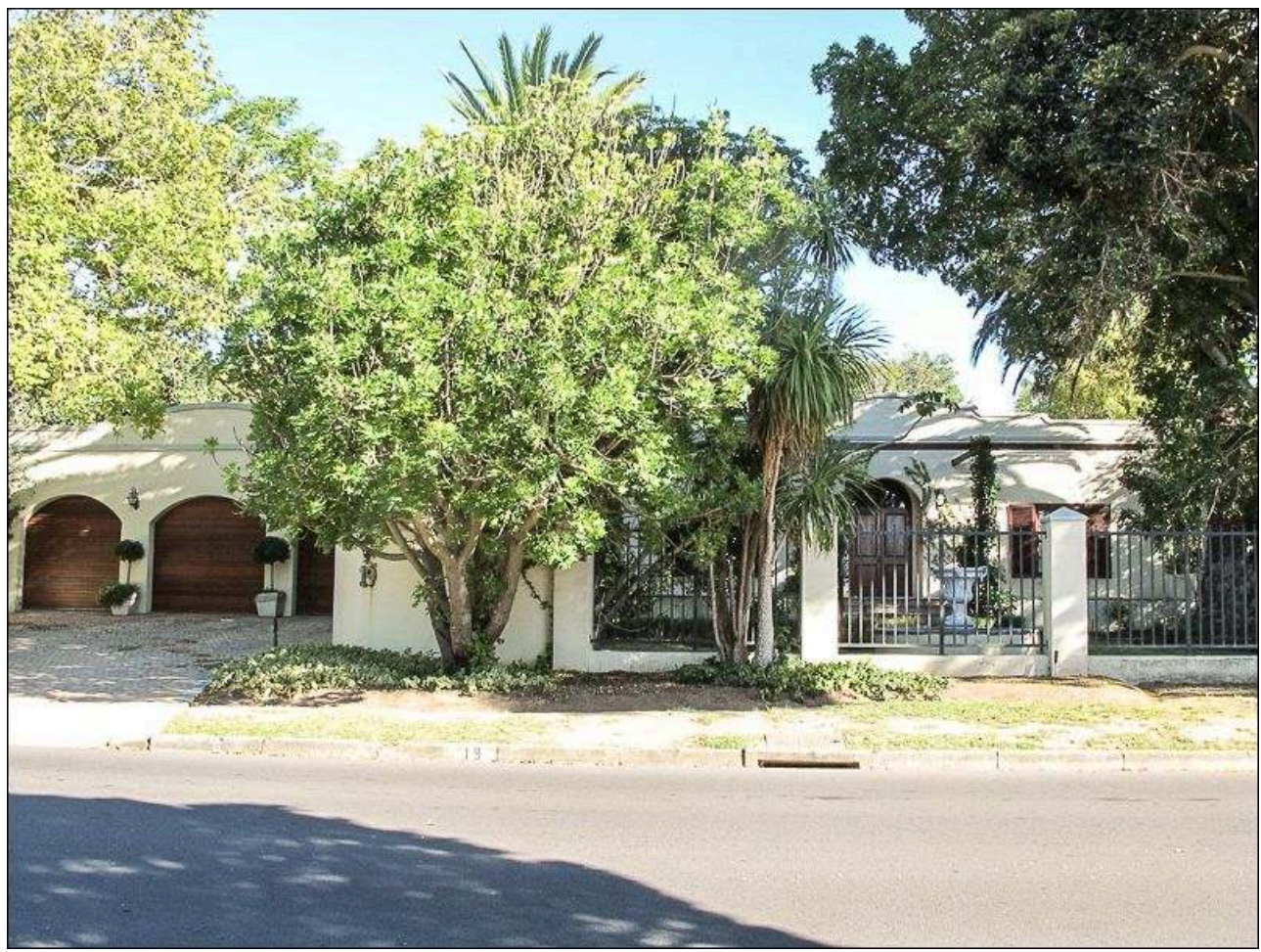

Durbanville : « Une maison style Durbanville [...] luxueuse, froide, sans caractère ». Grille, triple garage. Audi 97 de Christo, sa Renault Clio. Télécommandes, chiens jappant dans la rue. «Dans ce quartier, les chiens aboient sans arrêt, toute la journée. Dogville, c'était le nom qu'elle donnait à Durbanville » (Trackers).

Auteur : M. Berger, avril 2013. 
Illustration $6 b$ - Les banlieues Nord : Durbanville

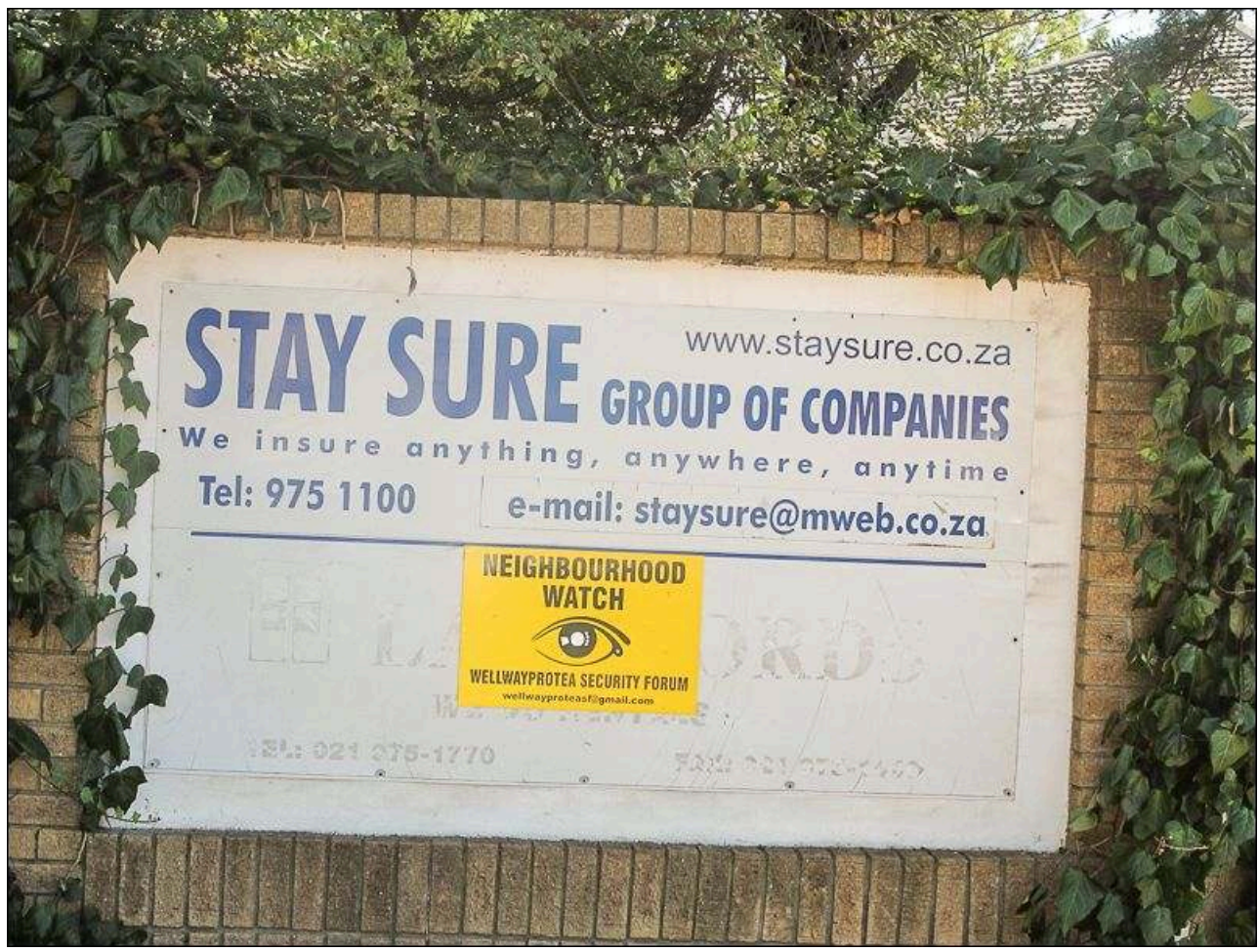

Auteur : M. Berger, avril 2013

Illustration 7a - Les banlieues Nord : Tygersberg

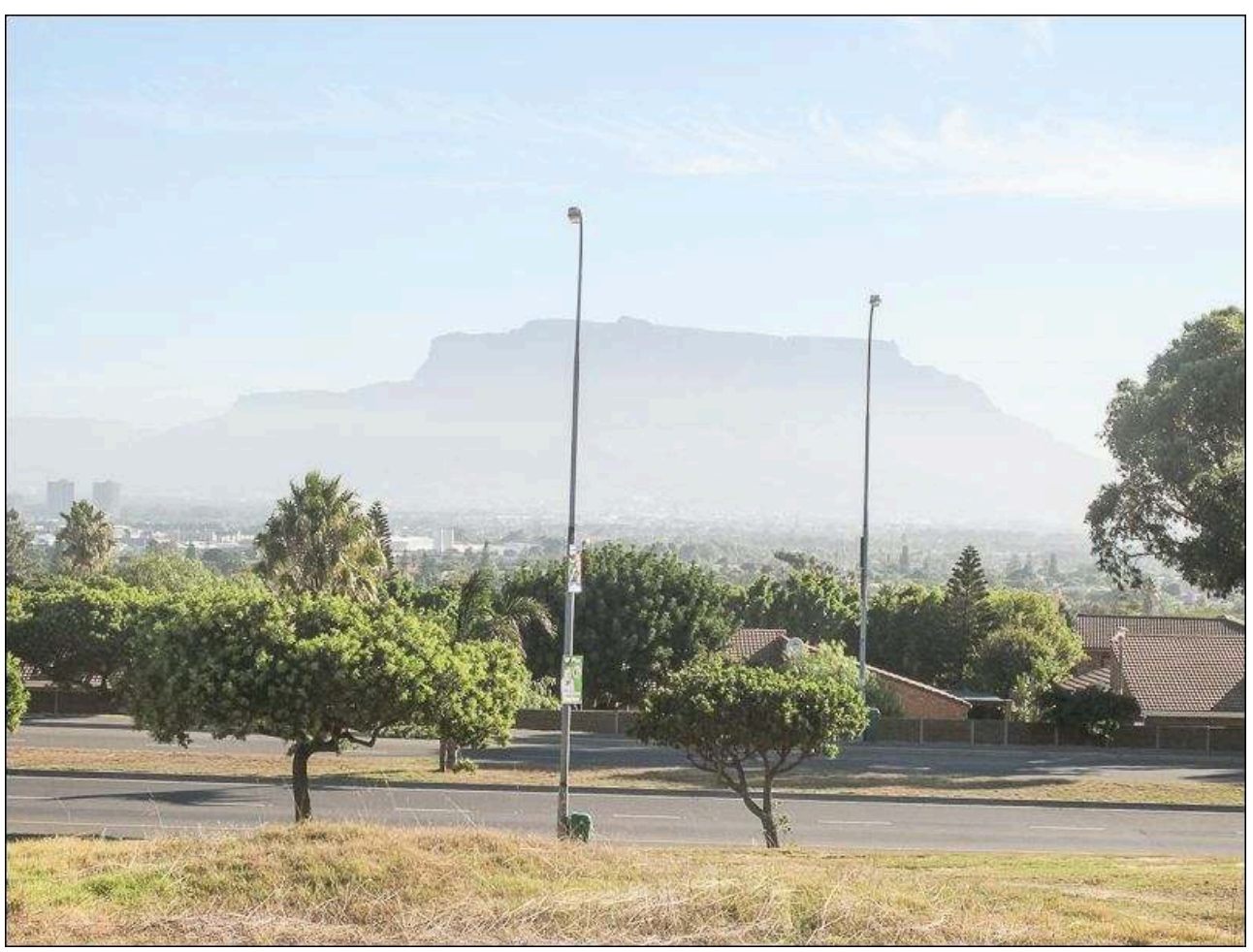

Auteur: M. Berger, avril 2014 
Illustration $7 \mathrm{~b}$ - Les banlieues Nord : Tygersberg

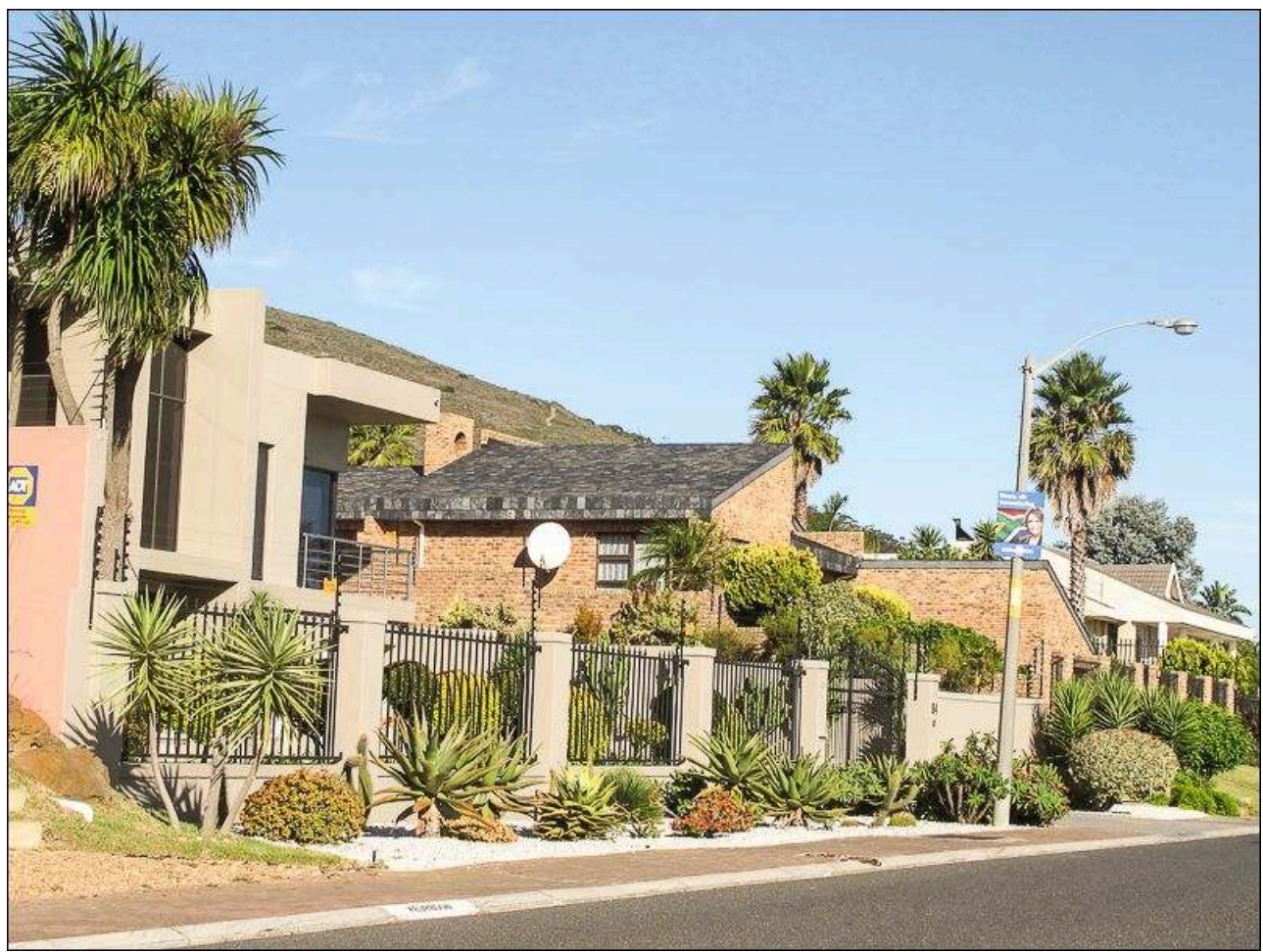

Tygersberg : où habitent Janina Mentz (directrice de l'Agence Présidentielle de Renseignement), Fransman Dekker (inspecteur de police coloured dont la femme a une belle situation), Olivier Nienaber (propriétaire d'une chaîne de salons de coiffure).

Auteur : M. Berger, avril 2014 
Illustration 8 - Les banlieues Nord : Milnerton Ridge

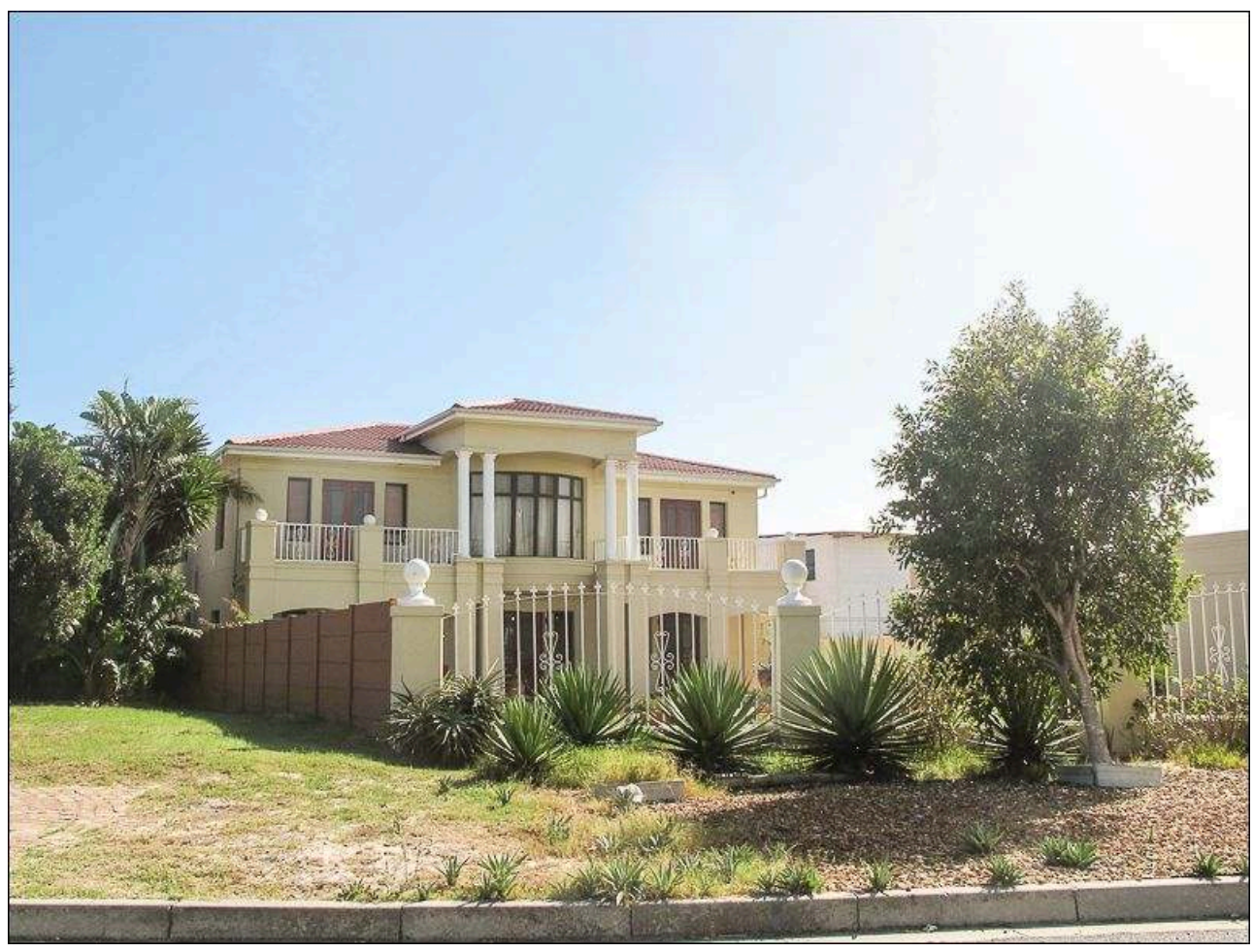

Milnerton Ridge : Une maison de style toscan du Transvaal, dans le quartier où habitent Orlando Arendse (baron de la drogue), Hope Beneke (jeune avocate) ainsi que Josh et Melinda Geyser, chanteurs de gospel sous contrat avec Afrisound.

Auteur : M. Berger, avril 2014 


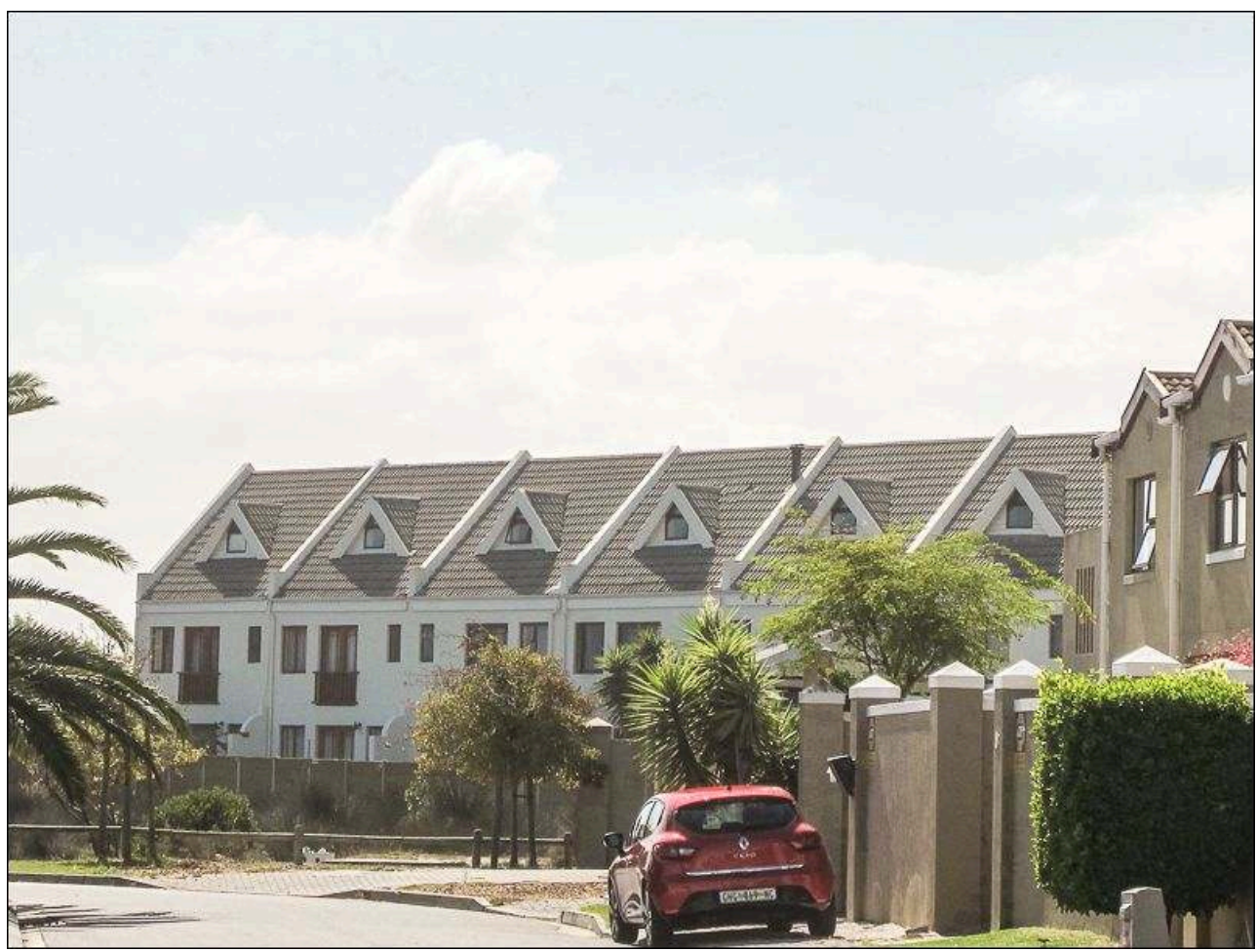

Parklands : « Il avait entendu parler de Parklands comme de Darklands, par référence à tous les Nigérians qui s'y étaient installés ces dernières années - encore que la majorité d'entre eux étaient de bons citoyens, titulaires d'emplois légaux ». « Il eut du mal à trouver la maison des Flint dans le labyrinthe de Parklands, où les habitations étaient toutes disposées en arc de cercle [..]. C'était un quartier nouveau, fruit de la spéculation immobilière. Les maisons serrées les unes contre les autres.. (Trackers)

Auteur: M. Berger, avril 2014.

Au sein même des banlieues de classe moyenne, de subtiles ségrégations se dessinent, par exemple entre les quartiers anciens de Parow ou de Goodwood, aujourd'hui moins cotés, où les inspecteurs de police cinquantenaires ont vécu leur enfance, et les nouveaux lotissements plus huppés, comme le quartier de Proteaville à Durbanville où résidait Milla Strachan avant son divorce, avec ses maisons «de style toscan du Transvaal ", mais parfois construits au rabais, dans le cadre d'opérations immobilières bâclées, avec des maisons serrées les unes contre les autres, comme à Parklands. Mais partout, dans ces quartiers de la classe moyenne blanche à majorité afrikaner, dominent l'entre soi des loisirs dominicaux autour de la piscine ou du braai, et l'enfermement sécuritaire, même si la couche de respectabilité ne résiste pas à l'examen.

\section{Travailler, se rencontrer et se distraire au Cap}

Si le City Bowl continue à concentrer l'essentiel des emplois et des lieux de rencontre des Capetoniens, la hausse des valeurs foncières et l'émergence de centralités (immeubles de bureaux, hôpitaux...) autour des malls récemment construits en périphérie redessine les territoires de la vie quotidienne des habitants de la métropole. Seules les plus grandes entreprises, avec les services financiers et de sécurité qui les accompagnent, ou les agences gouvernementales au plus haut niveau peuvent assumer 
les coûts de localisations dans le quadrilatère central, près des lieux du pouvoir politique, à Gardens. Si l'implantation des commissariats, comme le niveau et la qualification de leurs effectifs, suit celle des populations blanches, et se fait nettement plus lâche dans les townships, les bureaux des enquêteurs sont à Bellville, tandis que les PME louent des locaux dans les zones d'activité périphériques, comme le fait Tanya Flint, patronne récente d'une petite affaire d'entretien de piscines à Montague Gardens. On trouve tout dans les malls, dans la flânerie ou dans l'urgence ; on s'y donne rendezvous pour un repas ou un café, dans des chaînes où la mixité raciale, au moins dans les jeunes générations, devient la règle. Lieux de la drague des célibataires avant le weekend, il peut s'y échanger quelques tirs perdus, par exemple quand la police traque un tueur en série habitué du magasin Woolworth du Waterfront. Un maillage dense de centres commerciaux structure une offre de proximité dans les banlieues nord, avec des différences de standing reflétant le niveau social des quartiers voisins.

Petite classe moyenne à Panorama, que connaît bien l'inspecteur Benny Griessel, afrikaner natif de Parow, dont la carrière a longtemps piétiné par abus d'alcool pour conjurer l'horreur des scènes de crime; clientèle plus active à Tygervalley, que fréquente Milla Strachan, ex-épouse de cadre d'entreprise, habitant Durbanville, quand elle ne recoure pas à l'offre de proximité ; offre plus sélective à Canal Walk (Century City) où Margaret Joubert, ancienne habitante des banlieues sud, entraîne son mari ou son ex-collègue Griessel en quête de costumes élégants adaptés à leurs nouvelles fonctions.

\section{Illustrations 10a - Centre commercial du Waterfront}

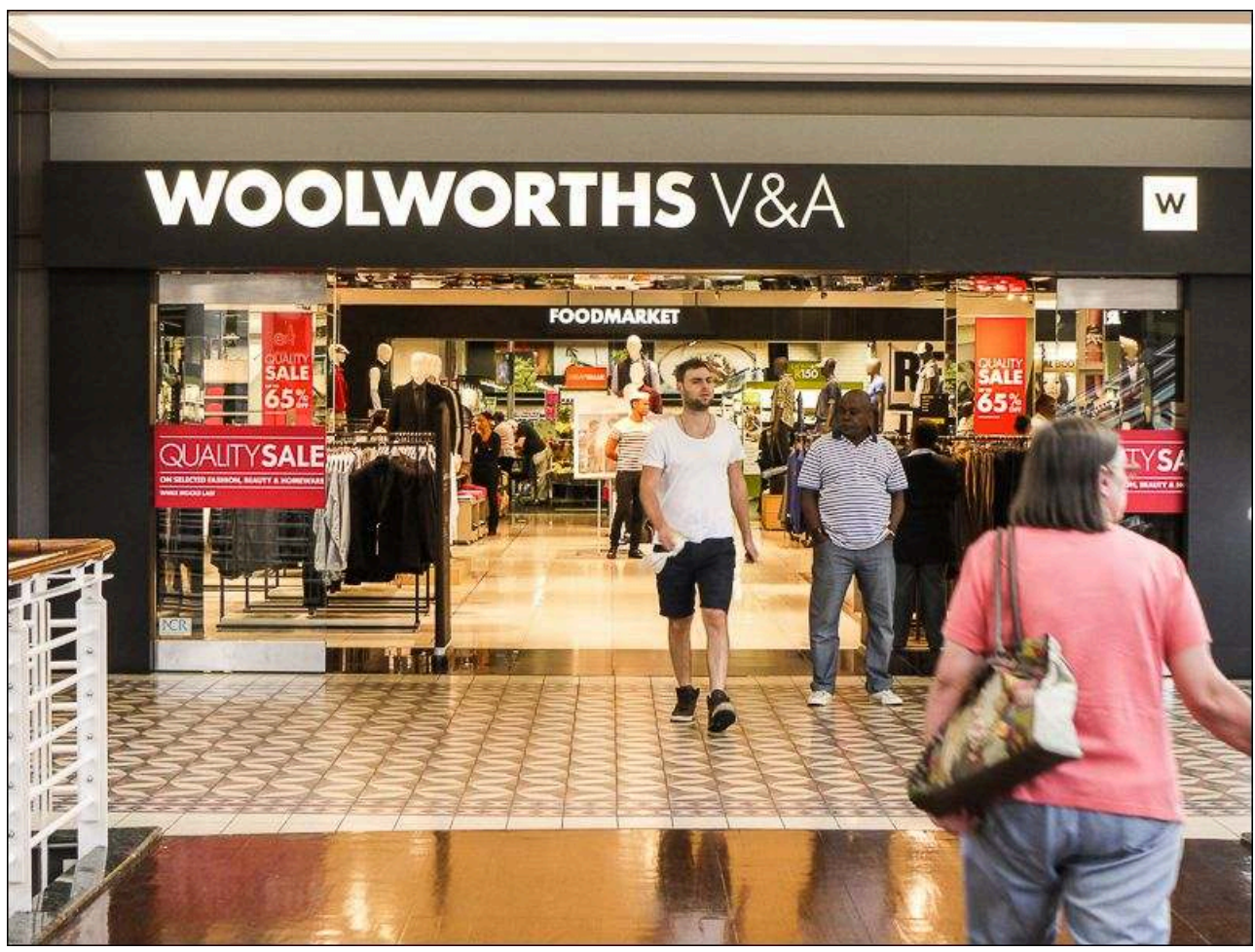

Auteur : M. Houssay-Holzschuch, avril 2014. 
Illustration 10b - Centre commercial du Waterfront

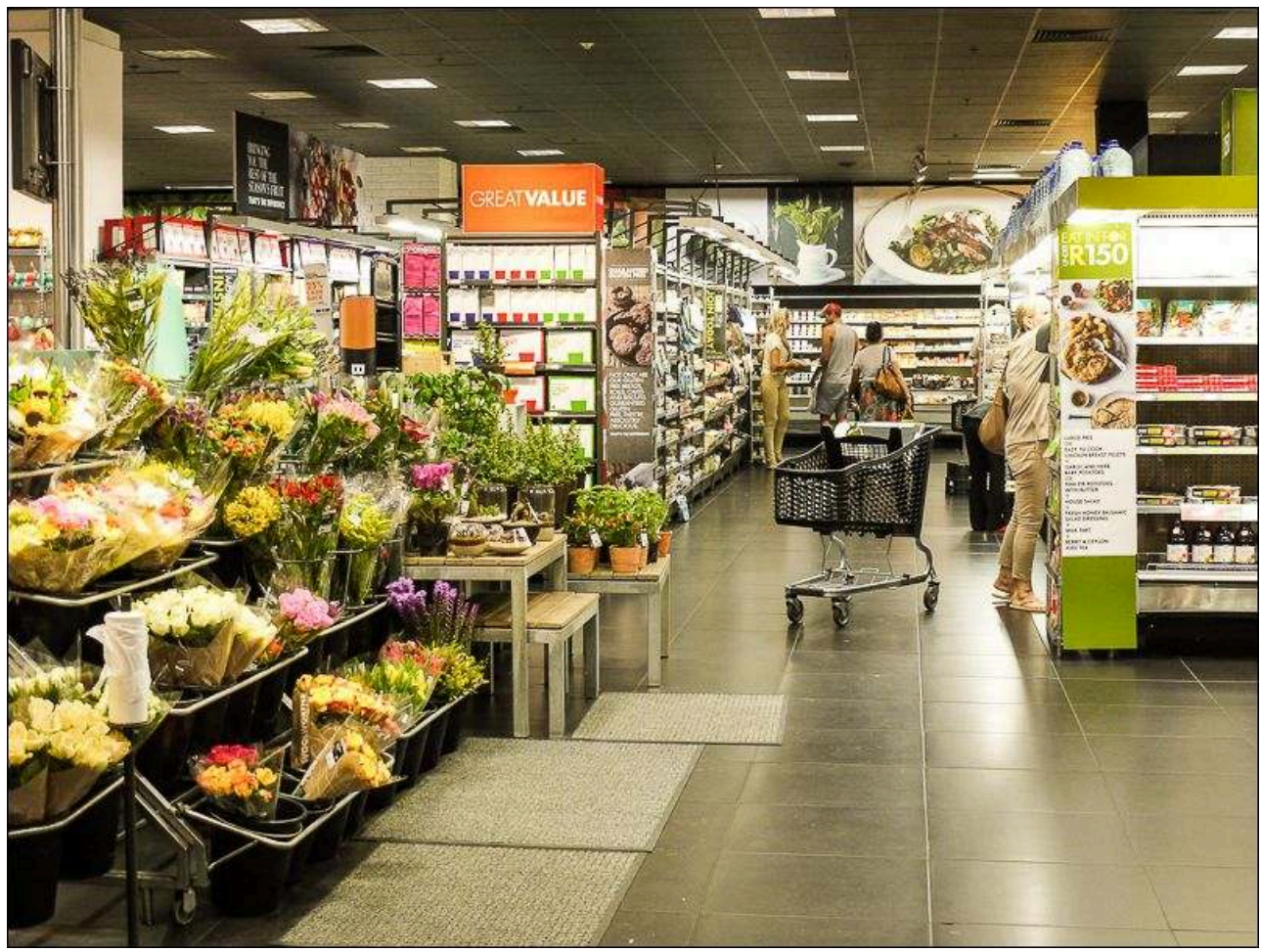

Le magasin Woolworth du WaterfronT, où un tueur en série drague ses futures victimes, le vendredi après-midi, au rayon alimentation. La police lui tend un piège, des tirs sont échangés au rayon des vêtements (Devil's Peak)

Auteur : M. Houssay-Holzschuch, avril 2014. 
Illustration 11 - Centre commercial de Century City

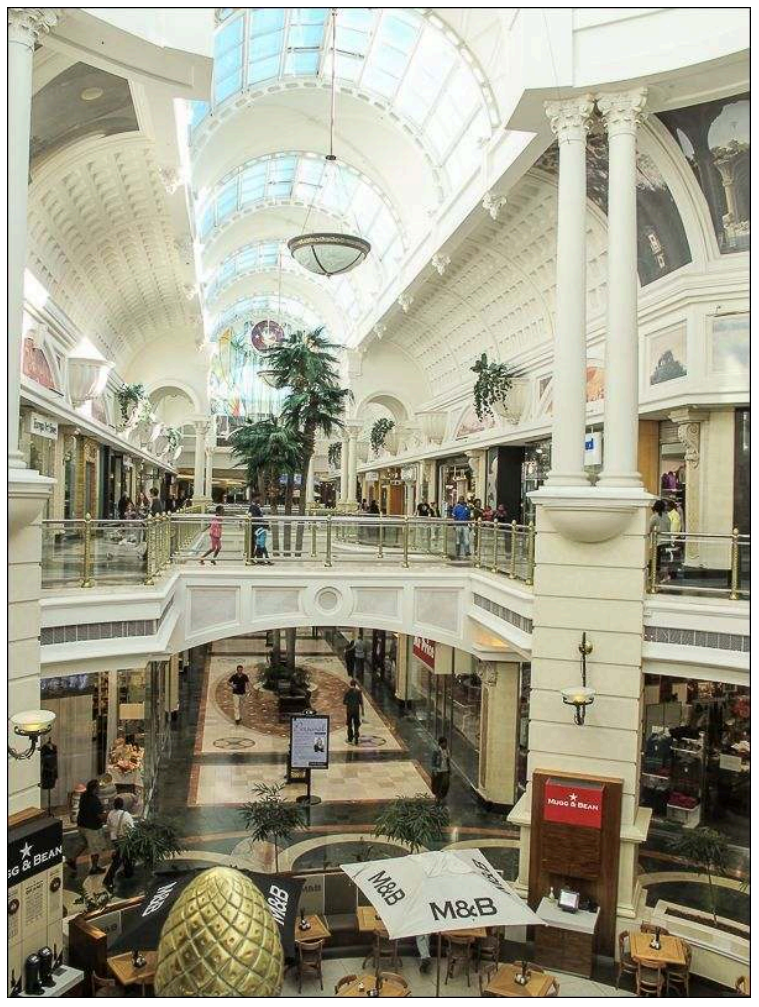

Canal Walk, à Century City : C'est là que les inspecteurs B. Griessel et M. Joubert viennent acheter leurs vêtements pour des occasions exceptionnelles (Seven Days).

Auteur: M. Berger, avril 2012.

Mais les rues et places du City Bowl gardent toute leur attractivité, pour ceux qui y travaillent comme pour les nombreux touristes qui s'y pressent. Loin du bureau et du regard des collègues, inspecteurs de police, détectives privés ou journalistes s'y rencontrent pour un bref échange, à Green Market square ou dans un des cafés de Long Street, mais il existe aussi des lieux plus sélects ou plus discrets, favoris des patrons de la police ou du renseignement, quand ils n'échangent pas leurs informations au cours de promenades dans les Jardins de la Compagnie. Lieu de concentration des commerces, mais aussi des auberges pour backpackers dans de vieilles maisons victoriennes, Long Street attire aussi, parfois à leurs risques et périls, les touristes étrangers, comme les deux étudiantes américaines résidant au Cat and Moose en feront l'expérience, au terme d'un safari africain. Car au Cap comme ailleurs, derrière la façade cool, tout est possible. 
Illustrations 12a - Long Street, dans le City Bowl

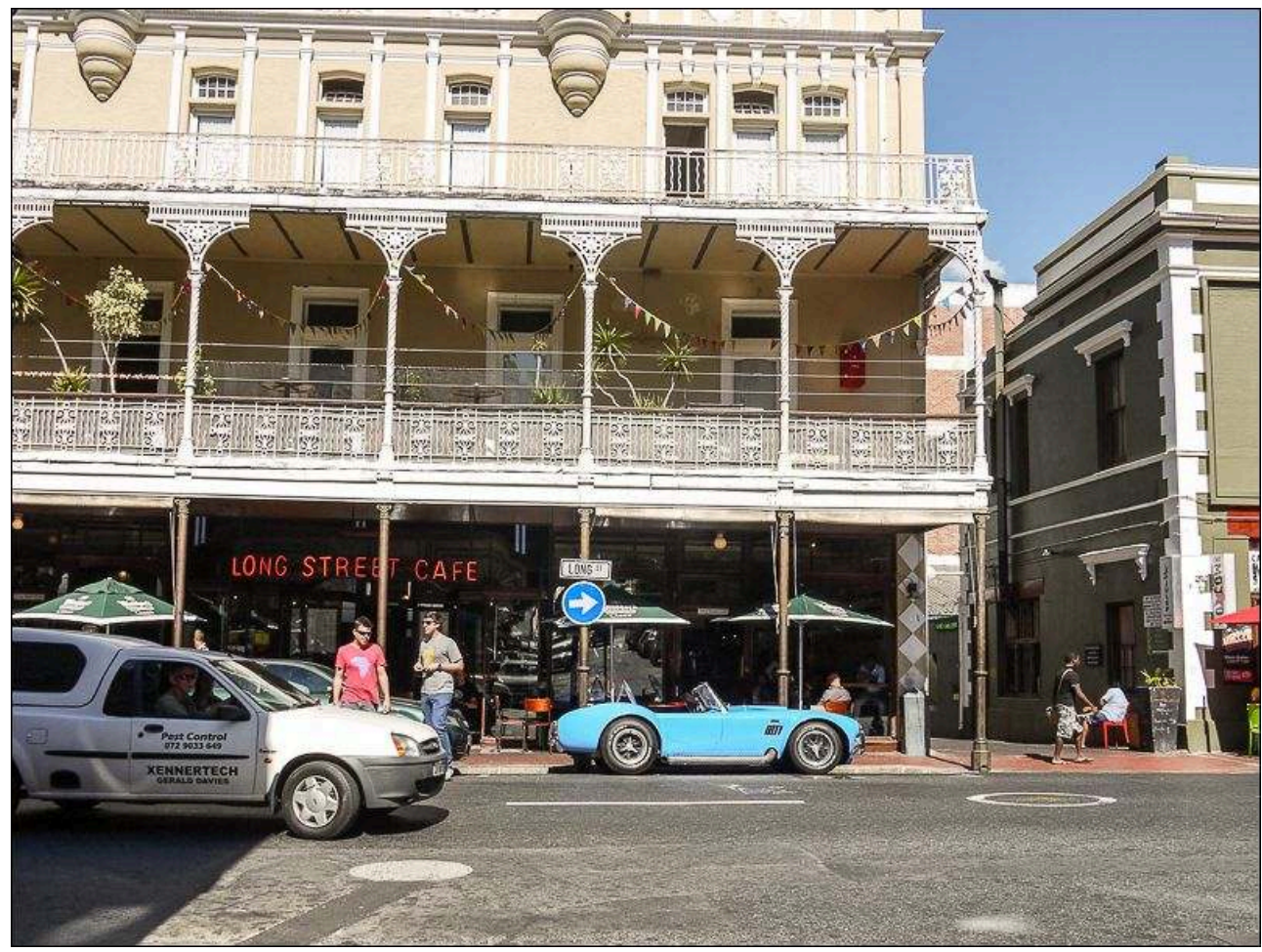

Le café où discutent, le soir, Janina Mentz et son chef et où déjeunent Hope Beneke, avocate, et KaraAn Rousseau, patronne d'un grand groupe de presse.

Auteur : M. Houssay-Holzschuch, avril 2014. 


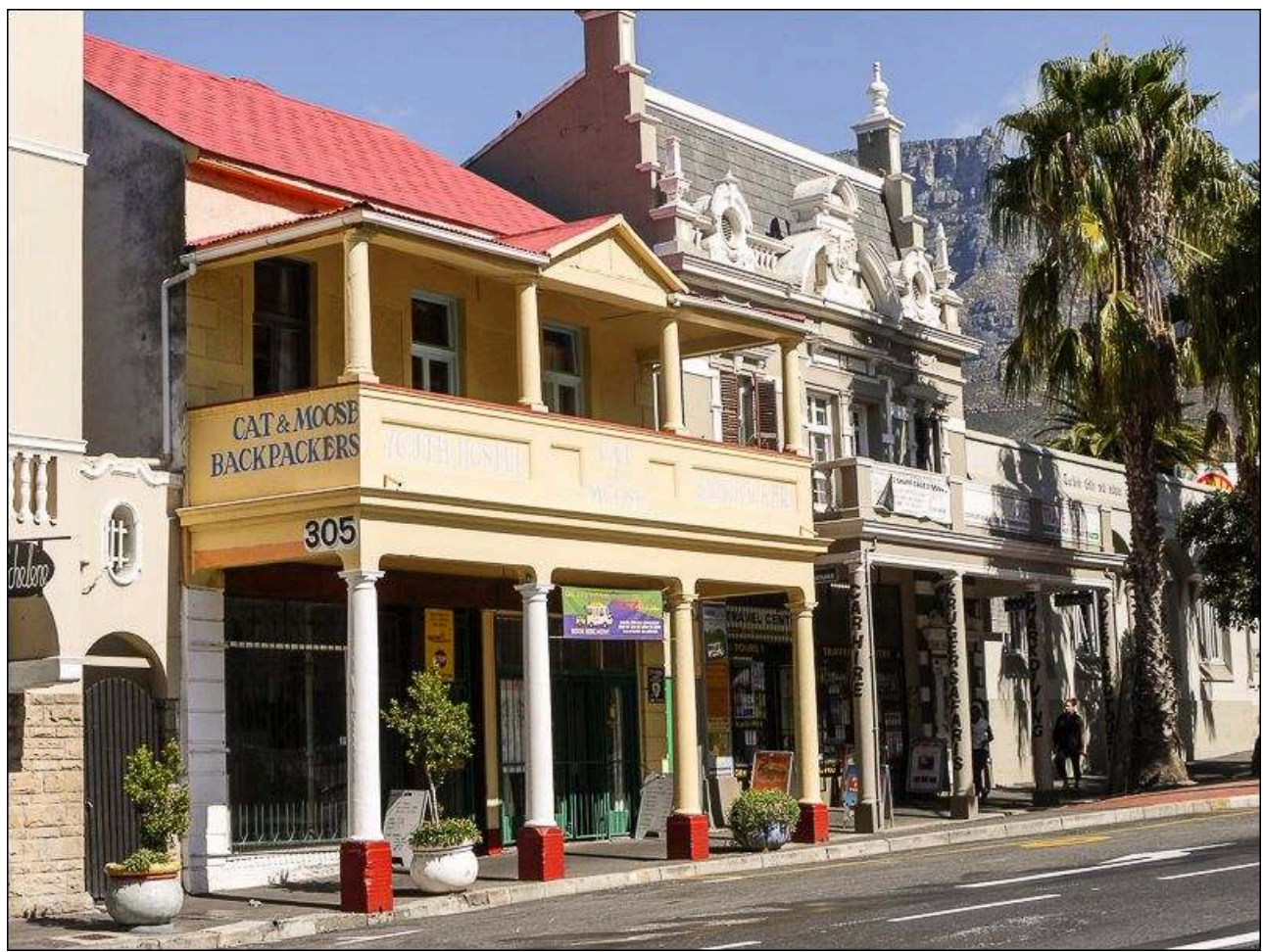

L'auberge (Backpackers) Cat \& Moose où résident deux étudiantes américaines. «Immeubles semivictoriens ne dépassant pas deux étages pour la plupart, et dont certains étaient à présent peints de couleurs chatoyantes, probablement pour plaire aux jeunes ». "La Mecque du tourisme de masse au Cap : jeunes gens, étudiants européens, australiens et américains à la recherche de logements peu coûteux » (Thirteen Hours)

Auteur: M. Houssay-Holzschuch, avril 2014.

\section{Les lieux des crimes et délits}

Comme le notait récemment Mike Nicol, autre auteur sud-africain de romans policiers ${ }^{14}$, le cadre topographique du Cap, entre océans et montagne, et la variété des quartiers réunis dans un espace rapidement parcouru grâce à un système dense d'autoroutes urbaines, se prêtent admirablement à la dramatisation nécessaire aux intrigues policières. Et Deon Meyer ne manque pas de rappeler l'excellence des infrastructures sud-africaines, comparée à celles des États voisins, constituant un facteur attractif pour les activités criminelles: un système bancaire efficace, des réseaux de télécommunications, routiers, ou aériens étendus, tout comme le recrutement possible de main d'œuvre dans les gangs des Cape Flats (Trackers, p. 106).

Pour autant, dans ses romans, les lieux des crimes et délits demeurent relativement circonscrits, peu évidents voire invisibles, lorsqu'il s'agit de la criminalité financière et de la corruption. L'auteur s'intéresse peu à la criminalité ordinaire, aux actions et formes répétitives telles les violences domestiques, qui gangrènent les townships.

Il les évoque rapidement dans Dead Before Dying: viol d'une fillette par un gang d'adolescents à Mitchell's Plain, meurtre par supplice du collier sur la plage de Macassar, assassinat d'une femme par son mari alcoolique, multiples vols à main armée tournant mal. Dans Devil's Peak, également, où se croisent la recherche d'un tueur en série assassinant des femmes seules d'âge moyen dans leurs appartements de Green Point ou Sea Point, et l'œuvre de justicier de Thobela Mpayipheli, qui 
recherche les tueurs d'enfants, violeurs ou parents indignes, pour venger le meurtre du fils de sa compagne, pris dans une fusillade dans une station-service. sont divers, tout comme les lieux où ils opèrent. Les bavures classiques à l'occasion de vols à main armée exploitant les failles dans les forteresses sécuritaires des quartiers de classe moyenne, les règlements de compte mettant en scène les mafias sud-américaines ou russes et des gangs liés au trafic de drogue constituent un bruit de fond occupant la police mais n'intéressent que moyennement Deon Meyer. Par contre, il s'attache souvent aux rencontres improbables entre personnes que tout semble séparer aujourd'hui, mais que rapproche un passé commun au temps de la Lutte, quel que soit le camp où ils se situaient alors, réactivant des solidarités anciennes ou liquidant d'anciens conflits. D'où la diversité des acteurs, des motifs et des lieux des crimes.

Du comptable injustement accusé d'un meurtre qu'il n'a pas commis et qui tire sur la police incapable de retrouver l'assassin de sa fiancée, aux gangs colombiens de la drogue retranchés dans de somptueuses maisons à Camps Bay, les scènes de crime et leur environnement urbain dessinent un large spectre de situations. Tout au plus peut-on repérer la commodité de no man's lands situés à la périphérie d'une ville qui est loin d'occuper la totalité de son territoire municipal : un club hippique un peu déglingué à Fisantekraal, au-delà de Durbanville; ou des terrains vagues proches d'anciens stands de tir de l'armée, aujourd'hui désaffectés, sur la route d'Atlantis. Mais la fusillade peut tout aussi bien éclater, avec ses dégâts collatéraux, au rayon vêtements du magasin Woolworth du Waterfront, ou chez Carlucci, ravissant café situé à un coin de rue du quartier résidentiel d'Oranjezicht.

int il est aisé de lire dans l'œuvre de Deon Meyer la géographie sociale des quartiers du Cap, autant il est difficile, à partir de ses romans, de dresser une carte des lieux du crime. Car tel n'est pas l'objet de l'auteur, plus soucieux d'offrir les cadres les mieux adaptés aux intrigues de ses thrillers, que de dresser un tableau des bas-fonds d'une métropole dont il aime à souligner qu'elle offre non seulement des paysages magnifiques, mais aussi une liberté d'action et des possibilités d'épanouissement personnel et social à qui souhaite rompre avec les anciens carcans, et qu'elle constitue un pivot, et un atout essentiel, dans la mondialisation, même s'il s'agit parfois de celle du crime...

\section{Le Cap, de la Mother City à la ville globale}

\section{Le Cap, ville-mère, ville insulaire et pôle d'attraction}

Première cité fondée en Afrique australe par et pour les Européens (HoussayHolzschuch, 1996 et 1999), la ville du Cap fait encore, d'une certaine façon, figure de ville insulaire, qui, si elle tisse ses réseaux vers d'autres pôles du territoire sud-africain, et apparaît (ou réapparaît) de plus en plus branchée sur des réseaux mondiaux, garde une forte spécificité et un pouvoir d'attraction considérable sur les forces vives du pays, et bien au-delà.

19 Son insularité, elle la doit, pour partie, à son amphithéâtre montagneux adossé à deux océans, qui isole la ville, ferme rapidement l'arrière-pays, à l'exception de quelques passages stratégiques par les cols dans les monts Hottentots Holland, cadre dont Deon Meyer souligne maintes fois la splendeur :

un panorama splendide, à couper le souffle, avec la baie bleutée et « la ville nichée au pied, confortablement [..] telle une enfant que la montagne enlace » (Thirteen 
Hours, p. 103). «Il aurait voulu se tourner vers Pakamile et lui dire : regarde, voici la plus belle ville du monde" (Devil's Peak, p. 69). Observée à l'aube depuis le Tygerberg, « la ville était tapie comme un cœur qui bat au ralenti, un cœur dont les artères serpentaient jusqu'à Groote Schuur et Observatory, Rosebank et Newlands, Khayelitsha et Gugulethu en direction de Kraaifontein, Stellenbosch et Somerset West. Riches et pauvres côte à côte dans le sommeil, un géant en repos » (Heart of the Hunter, p. 465).

Mais ce n'est pas seulement au sens propre que la ville brille de mille feux : elle attire, dans l'ensemble du territoire sud-africain et au-delà, dans l'Afrique australe, une jeunesse soucieuse de s'éduquer et de réussir, mais aussi de se libérer des anciennes règles et des anciens carcans, dans une société plus ouverte et plus moderne. Ceux qu'étouffe la morale austère du Free State, en premier lieu les femmes, condamnées trop souvent, dans le Nord rural et ses petites villes très conservatrices, au rôle d'épouses au foyer dociles et ternes, cachant une double vie derrière une façade de respectabilité.

C'est le cas de la mère de Christine van Rooyen (Devil's Peak). Zatopek (Zet) van Heerden, après une formation à l'École de police à Pretoria s'ennuie ferme dans son premier poste de policier en tenue dans un commissariat du Gauteng où il passe son temps à recueillir des plaintes de voisinage ou de disputes conjugales, des vols de voiture, des petits délits de cols blancs. Il reprend des études de criminologie, fait un stage au FBI en Virginie, obtient un poste d'assistant au département des sciences de la police à l'UNISA. Pour échapper à la routine d'un doctorat et à un mariage conventionnel, il accepte un poste au bureau des homicides, au Cap. Sa mère, Joan van Heerden, artiste peintre, fille d'une actrice et d'un professeur de théâtre de Pretoria, s'y installe près de son fils, après des années passées à Stilfontein, une petite ville minière près de Potchefsroom ${ }^{15}$. Connue et admirée dans différents milieux (avocats, patrons de presse), elle y trouve un marché pour ses tableaux (Dead at Daybreak).

Bon nombre de personnages des romans de Deon Meyer, natifs du Nord (Free State, Gauteng), venus faire leurs études dans la très conventionnelle université de Stellenbosch, ont souhaité rester au Cap, symbole de libération des mœurs (gay friendly) et de tolérance, pour échapper à " la main de fer de l'Église réformée néerlandaise ", au modèle parental d'ennui conjugal et d'obéissance, d'hypocrisie derrière le sens du convenable et du devoir.

C'est le cas de Christine van Rooyen, devenue call-girl pour élever sa fille, et de son amie Vanessa (Devil's Peak). C'est aussi celui d'Emma Le Roux, dont les parents sont devenus des industriels prospères dans le Gauteng grâce aux commandes de l'armée; elle travaille en free lance dans le marketing publicitaire, et a très bien réussi dans son métier : petite maison à Oranjezicht, voiture sportive (Blood Safari). Ou celui de Hope Beneke, avocate qui vient de fonder son propre cabinet avec une associée, et d'Alexa Barnard, fille d'un petit cadre de banque : elle a commencé sa carrière de chanteuse à Johannesburg, avant d'être découverte par Adam Barnard, avocat qui se lance dans la pop music et fait d'elle une star, avant qu'elle ne sombre dans l'alcool.

\section{Le Cap versus Johannesburg}

Les échanges entre Gauteng et Western Cape tels que les décrit Deon Meyer ne se limitent pas aux trajectoires migratoires de ses personnages. Ils renvoient aux spécialisations des villes dans le système urbain sud-africain, et à leurs positions respectives dans la mondialisation, $\mathrm{y}$ compris celle du crime. C'est dans le Gauteng, près du pouvoir politique, que les industriels de l'armement font fortune ; mais c'est au Cap 
que la SCA (Southern Cross Avionics), qui fabrique des systèmes de guidage pour missiles et avions de chasse, y compris pour Dassault, emploie, dans ses bureaux de Century City, 500 personnes dont 50 ingénieurs high-tech. C'est à Pretoria que se situent l'École de la police, ses services spécialisés dans les expertises les plus pointues, le haut commandement. Si Le Cap, de par sa situation portuaire, constitue une des portes d'entrée des mafias du crime, c'est à Johannesburg que les plus gros poissons résident (à Sandton) et font leurs affaires : entre les deux villes, les allers et venues sont incessants, pour conclure des deals, avec comme annexes Windhoek et Walvis Bay en Namibie, arrière-pays traditionnel de l'Afrique du Sud, et pays dont les frontières sont réputées particulièrement perméables.

Pour les parents de Carina Oberholzer, fermiers dans le Nord-Ouest, Johannesburg est une ville trop dangereuse pour une jeune femme : ils pensent - à tort - que leur fille est plus en sécurité au Cap, alors que leur fils peut affronter Johannesburg.

Compte tenu de la tertiarisation de son économie, Le Cap est « une Mecque pour le célibataire des classes moyennes, la proportion hommes-femmes y étant plus intéressante d'un point de vue statistique " (Dead at Daybreak, p. 379). Mais c'est aussi une ville, « où on garde ses distances, où l'on remonte le pont levis chaque soir, où l'on reste entre soi et soi ", contrairement aux bourgs du Karoo où réside désormais Lemmer, avec leur sociabilité traditionnelle de voisinage. Même si, lors les braai du samedi soir dans les banlieues nord, la bière Castle et l'alcool coulent à flots autour des barbecues où les hommes s'occupent de la cuisson de la viande, tandis que les femmes bavardent à la cuisine (Dead Before Dying). Car Le Cap, c'est aussi une ville relax, raillée par les jaloux du Gauteng, et le leur rendant bien.

\section{Une ville où il fait bon vivre, qui est aussi une ville créative}

24 Le Cap est une ville où on est bien : grand ciel bleu, juste ce qu'il faut de vent pour rafraîchir l'atmosphère et dissiper les nuages qui enveloppent de leur couronne la Montagne de la Table. Il y pleut rarement: un seul des romans de Deon Meyer s'y déroule en hiver, mais aux journées froides et venteuses, où il pleut à verse, succèdent « des jours de grand soleil entre des fronts froids, clairs comme le verre [..], joyaux qui brillent sur la robe sombre de l'hiver» (Dead at Daybreak, p. 163). L'été, il n'y fait pas trop chaud, le corps est à l'aise, on ne sue pas comme dans la chaleur humide du Kruger, sous le soleil implacable du Limpopo aux arbres rabougris, ou dans la fournaise $\mathrm{du}$ Karoo, dont les paysages aux vastes horizons sont pourtant superbes. Au Cap, on soigne sa forme en faisant du jogging sur les plages ou dans les salles de sport de Virgin Active. Tout est fait pour le confort d'un corps mis en valeur par des vêtements seyants: c'est là qu'on vient s'habiller, là que se crée une mode décontractée et élégante, branchée sur l'Europe. Les bourgeois du Gauteng y débarquent avec femmes et enfants pendant les vacances de Noël ou au début janvier :

«Des enfoirés suffisants qui se voyaient comme un don du ciel pour la ville du Cap ", "mères faisant du shopping comme des furies ", "parvenus arrogants qui avaient attendu pour se remplir les poches durant les fêtes de Noël à Sandton et se ramenaient ensuite pour leur crise de dépense annuelle » (Thirteen Hours).

$25 \mathrm{Au}$ Cap, ville créative, ville internationale, on accueille, outre les investisseurs étrangers nombreux à être attirés par des affaires faciles, les pop stars et les acteurs du monde entier, en tournée ou en tournage. 
Une bonne clientèle pour l'agence haut-de-gamme Body Guard de Jeannette Louw (la patronne de Lemmer), autrefois adjudant-chef du Collège féminin de l'armée, qui attend de ses employés loyauté et professionnalisme, et ne cache pas qu'elle aime consoler les femmes esseulées. La photographe d'art Anni de Waal, qui a un studio à De Waterkant Village, préfère quant à elle être payée en euros par ses clients des magazines de mode européens.

Les studios de publicité, les artistes et artisans free-lance, s'installent dans les nouveaux lieux branchés mais ont souvent du mal à trouver un travail à plein temps : «Maintenant, c'est assez à la mode de venir crever de faim au Cap quand on a un tempérament artiste » (Dead Before Dying, p. 355). Comme le souligne Myriam HoussayHolzschuch (2010, p. 7).), «Cape Town aspire donc à devenir une ville-relais de la mondialisation, une ville globale secondaire. D'un point de vue économique, la volonté d'arrimer le développement local à l'émergence globale aboutit à l'exact contraire de ce que l'on cherchait, puisque cela accroit les inégalités et la polarisation sociale ».

\section{Blancs, Blacks, Coloureds à l'heure de la nation Arc- en-ciel}

\section{Un passé qui ne passe pas?}

La publication des romans de Deon Meyer (dans leur version originale en afrikaans) s'étage entre 1996 et 2011, embrassant une période cruciale pour l'Afrique du Sud, de la présidence de Nelson Mandela à celles de Thabo Mbeki, puis de Jacob Zuma. Au fil des pages, les commentaires sur l'évolution politique et sociale de l'Afrique du Sud ne manquent pas, mais s'ils rendent compte de la diversité des points de vue, allant de l'espoir à la désillusion, ils sont toujours nuancés.

Deon Meyer retrace, par exemple, le parcours de Hope Bennett (Dead at Daybreak, p. 208-209) : autrefois (avant 1992), elle a voté pour le Parti National, elle a cru à l'apartheid, comme ses parents, amis et professeurs, à Bloemfontein. Elle croyait à ce qu'on disait à la radio, et dans le journal afrikaans du coin. Elle croyait les noirs incultes et voués aux tâches ancillaires. Elle a lu une biographie de Mandela et changé d'avis: «Vous savez ce que ça fait [...] de voir ses idées, ses amis, ses parents, ses chefs, son passé, son histoire, sous un jour complètement différent? [...] De s'apercevoir que tout ce à quoi on croyait et on avait foi était faux $[. .] ».($ p. 208). «Je suis arrivée à ouvrir mon esprit à la vérité. À voir clair après avoir été si longtemps aveugle [...]. Je me suis juré de chercher la vérité [...]. Plus question de juger les gens sur leur couleur, leurs croyances ou leurs actes [...]. ». La période de transition a été difficile: "C'était une époque bizarre. C'était ... Découvrir tout à coup ce que l'ennemi avait sur vous, leur montrer ce que nous, on avait, c'était surréaliste. L'ennemi n'était plus l'ennemi, après toutes ces années ». «On était conditionnés, c'était ancré en nous depuis si longtemps, les secrets, l'idée que c'était eux contre nous " (Heart of the Hunter, p. 149).

Tous admettent que les choses ont changé, qu'ils ont changé, mais cela ne suffit pas, n'est pas allé assez loin. En 2001, la déception commence à être forte, concernant la nation arc-en-ciel, les difficultés de la reconversion ou de l'amalgame.

Selon Orlando Arendse, qui a pourtant très bien réussi comme baron de la drogue avant l'arrivée des Nigérians, la liberté ne suffit pas, le township est toujours un ghetto, la pauvreté demeure : «La liberté ne nourrit pas son homme. On ne peut pas acheter une voiture et une maison avec. [..] Madiba était notre Moïse et il nous a emmenés jusqu'à la terre promise mais il n'y avait ni lait ni miel » (Heart of the Hunter, p. 255). 
corruption est là, bien présente dans les opérations juteuses permises par la législation $\mathrm{BEE}$, dans lesquelles se côtoient cabinets juridiques ayant pignon sur rue, riches intermédiaires scrutant les opportunités sur le marché, anciens de la Lutte devenus ministres adjoints puis dirigeants syndicaux utilisant l'argent des caisses de retraite des mineurs à leur profit, anciens agents du KGB et soldats démobilisés de Tchétchénie, hommes de main au service d'oligarques réfugiés à Londres flairant les bonnes affaires (Seven Days). Il reste que tout n'est pas noir, que des progrès considérables ont été accomplis, comme le précise Deon Meyer dans un entretien au moment de la sortie de Trackers: "Les romans policiers fournissent surtout une vision du côté obscur des villes et pays. C'est là mon seul véritable regret, en tant qu'auteur de policiers. Parce que l'Afrique du Sud, ce pays que j’aime passionnément, est très différente de cette image sombre et limitée qui émane de mes livres. J’ai une vision très positive de l'Afrique du Sud, et de l'avenir de mon pays. Bien sûr, nous avons de problèmes à résoudre, tout comme le reste du monde. La pauvreté est définitivement notre plus gros challenge à relever, et l'éradiquer serait peut-être la clé de notre réussite. Mais nous faisons tout de même d'énormes progrès. [...] » ${ }^{16}$. Des progrès accomplis, et de ce qui reste à faire, la police est sans doute un bon exemple.

\section{La police, une micro-société témoin?}

30 La période décrite par Deon Meyer est marquée par des changements de fond dans l'organisation, le recrutement et les méthodes de la police, qui, s'ils déstabilisent les anciens, contribuent à la rapprocher des citoyens. Changement de nom (le SAPS ${ }^{17}$ ), de grades (avec l'abandon, puis le retour, aux hiérarchies militaires); de structures, pour tenter de s'adapter aux nouvelles demandes et aux nouvelles formes de criminalité ; de méthodes, pour respecter les droits des citoyens et s'adapter aux nouvelles technologies d'investigation; mais surtout, changements majeurs dans la composition raciale des effectifs, avec une politique systématique de discrimination positive visant à recruter et promouvoir des policiers Noirs, xhosas (sous Mandela et Mbeki), zoulous (avec l'arrivée au pouvoir de Zuma), mais aussi tswanas, sothos, et locuteurs des différentes langues officielles du pays, dans la mesure où Le Cap attire des immigrants de l'ensemble des provinces, et bien au-delà.

Dans la police comme dans l'armée, le renouvellement des hommes est important. En 1996, on ne compte qu'un seul inspecteur coloured à la brigade des vols et homicides, dont le chef, Bart de Wit, un Blanc ayant accompagné l'ANC en exil, vient d'être nommé par le nouveau ministre de l'Intérieur, qui est Noir. Il affirme clairement qu'il s'agit de marquer la Nouvelle Afrique du Sud, avec le changement dans les façons de faire de la police: "On se remet comme il faut ou on se démet. Dans les communautés défavorisées, il y a des tas de gens qu'il faut absolument promouvoir ». " Tout le monde nous attend au tournant. Les Blancs aimeraient beaucoup que les Noirs commettent assez d'erreurs [..]» (Dead Before Dying, p. 30-31). Nombre d'anciens policiers Blancs afrikaners sont mis à la retraite avec des indemnités confortables leur permettant de fonder les nombreuses officines de sécurité qui se créent alors pour répondre à la montée de la criminalité et à la demande de protection des diplomates et hommes d'affaires étrangers qui reviennent en nombre en Afrique du Sud avec la levée des sanctions.

EchoGéo, 28 | 2014 

est chargé par le général John Afrika, coloured, de former une équipe de jeunes inspecteurs : 5 noirs (dont une femme zouloue), un coloured, pas un seul Blanc (Thirteen Hours). Presque tous les anciens policiers blancs, gros mangeurs, gros buveurs, pas très propres, sont en retraite, morts ou partis par lassitude, ayant attendu en vain une promotion impossible, comme Mat Joubert, qui avait cru à un nouvel idéal de police nationale effaçant les injustices du passé: «Ce bel outil avait été lentement empoisonné par la politique et les bonnes intentions sans suite [..], et pour finir, par l'avidité et la corruption » (Trackers, p. 564).

Tout le haut commandement des services de police est désormais Noir ou Coloured. Les nouvelles recrues sont soucieuses de bien faire, ont des tenues et une allure impeccable (contrastant avec le débraillé fréquent chez certains de leurs aînés afrikaners), mais sont encore inexpérimentées, se débrouillent mal sur le terrain, connaissant souvent mal la langue et la culture de ceux qu'elles sont censées protéger. Mais policiers et juges font plutôt bien leur travail, surtout quand ils sont noirs et doivent faire leurs preuves, à quelque échelon hiérarchique que ce soit. C'est moins net pour les coloureds, plus anciennement recrutés et promus, désormais bloqués par le système de discrimination positive : ils ont la réputation d'être arrogants, de ne se préoccuper que de leurs congénères et ne résistent pas toujours à la tentation de profiter des avantages de leur position, voire de se laisser corrompre, comme certains des anciens policiers blancs de la brigade des homicides qui a été dissoute. Mais les corrompus sont finalement rares, et les policiers transgressent peu les lois : s'ils prennent quelques libertés avec le droit, c'est pour accélérer les enquêtes. De même il y a peu de bavures, et elles sont le fait du monde de l'espionnage, de l'Agence Présidentielle de Renseignement.

Le policier, comme le garde du corps privé, n'est pas à l'aise avec les riches : par ses origines sociales, il n'appartient pas à la classe qu'il est amené à défendre et protéger, mais à une petite classe moyenne qui vit le plus souvent dans des maisons très moyennes des banlieues nord. Comme le reste de la société, la police est traversée par des conflits de classe, et de race.

Vusi Ndabeni, inspecteur noir (xhosa), se demande si la jeune et jolie Tiffany October, médecin légiste coloured, accepterait de boire une bière avec un Noir, fûtil inspecteur de police: il saute finalement le pas, avec succès (Thirteen Hours). À l'inverse, Fransman Dekker, coloured, le seul parmi les jeunes inspecteurs formés par Griessel, se sent menacé par les nouveaux recrutements de policiers noirs : «Ils mettent les négros en avant. On doit les caser partout ». « J'suis là pour remplir leur putain de quotas! T'as regardé le commissaire, jeudi ? Il n'avait d'yeux que pour ces foutus Xhosas, il ne m'a même pas vu. Huit pour cent de métis. Huit pour cent, nom de Dieu! " «Pas assez noir, désolé mon frère, dégage de là, trouve-toi un job chez Coin Security, va conduire un fourgon blindé » (Thirteen Hours, p. 236).

\section{Des races, et des classes}

En 1996, dans le premier roman publié par Deon Meyer (Dead Before Dying), l'inspecteur de police Mat Joubert a une femme de ménage xhosa, dont le mari vit à Soweto, les deux enfants dans le Transkei. En 2001, Miriam Nzululwazi, xhosa, employée subalterne à la succursale d'ABSA d'Heerengracht (dans le City Bowl), vit dans une petite maison à Gugulethu, qu'elle a achetée avec ses économies, et Thobela vient d'acquérir une petite ferme dans les collines du Transkei. En 2007, Fransman Dekker, Coloured comme son 
épouse, habite à Tygerberg, dans le même quartier que Janina Mentz, Blanche Afrikaner, chef de l'Agence présidentielle de renseignement.

Si les inégalités sociales se sont accrues, elles traversent tous les groupes raciaux.

Lemmer, fils d'un mécanicien afrikaner de Sea Point et d'une "anglaise» de Rosebank, déteste les riches afrikaners: "Ils se vautrent dans leurs immenses maisons derrière de hauts murs et des systèmes d'alarme, tout en se gavant de traiteurs de luxe devant les écrans plats des télés HD, avec une Mercedes ML, deux quads, une Harley et un hors-bord dans leurs garages triples». «Ils restent là comme des vautours à guetter, et à chaque faux pas du gouvernement, ils braillent « je vous l'avais bien dit ». Ce sont des racistes qui n'ont pas le courage de l'avouer » (Trackers, p. 223-224).

Les Coloureds, qui constituent la majorité de la population capetonienne, ont du mal à se situer:

Natasha Abader (assistante d'Adam Barnard, producteur de disques), à qui Fransman Dekker reproche d'être devenue bêcheuse en travaillant avec les Blancs, lui réplique : «Vous voulez qu'on soit une tribu séparée, nous les métis [...] Si vous ne vous intégrez pas, personne ne le fera pour vous " (Thirteen Hours, p. 326). Pour Mbali Kaleni, inspectrice zouloue, féministe et grassouillette, adepte des fast-foods : "Voilà le problème dans ce pays [..], toutes ces minuscules différences culturelles : une zouloue mange quand elle doit manger, c'est normal, naturel et il n'y a pas de quoi en faire un plat » (Thirteen Hours, p. 345).

Faut-il pour autant désespérer, face à ce cloisonnement, racial et social, à l'image de celui de la nation? Un vieux professeur d'université qui vit à Oranjezicht écrit un livre sur la reconstruction de l'Afrique du Sud après la guerre des Boers, pour que les Afrikaners se rendent compte qu'ils ont eux aussi traversé des épreuves, ont été opprimés, sans terres, écrasés comme les Noirs aujourd'hui, et se sont relevés grâce à la discrimination positive dans l'administration et l'économie, mais ont été ensuite corrompus par le pouvoir. "Nous sommes un pays qui a du potentiel, avec des gens bons et merveilleux qui ne veulent tous qu'une chose: un futur pour nos enfants" (Thirteen Hours, p. 320). Et Lemmer observe dans un restaurant des jeunes de 20 ans qui s'amusent: «Blancs et Noirs mêlés, décontractés, comme si notre pays n'avait jamais eu d'histoire ", "Il en va de même dans les centres commerciaux et les rues, comme si la ville représentait ce que pourrait être notre pays si l'on parvenait à effacer l'ombre noire de la pauvreté » (Trackers, p. 307).

Il n'est pas étonnant que l'Afrique du Sud, et tout particulièrement Le Cap, s'inscrivent dans le panorama des hauts-lieux de la littérature policière, compte tenu du niveau élevé de la criminalité, la violence étant à la fois héritée de l'apartheid, entretenue par les inégalités croissantes de richesse et l'existence d'un réservoir de jeunes sans travail. Dans les premiers romans, les références aux années de la Lutte et des opérations spéciales de l'armée sont encore très présentes. Au fil du temps, au fur et à mesure que l'on s'éloigne du tournant de 1994, les références aux conflits hérités se font plus rares, mettant au premier plan les violences ordinaires, la délinquance en col blanc, l'intrusion des réseaux internationaux du crime. Il n'est pas surprenant non plus que deux des auteurs de ce pays ayant accédé, dans le genre policier, à la renommée internationale, soient d'anciens journalistes. Mike Nicol $^{18}$ a souligné comment le passage à la littérature permettait, mieux que le flot des articles de presse rédigés dans l'urgence, d'aller au fond des choses, de condenser la réalité dans une intrigue romancée pour tirer la leçon des évènements. 

qu'il déploie, Deon Meyer brosse un tableau souvent saisissant de sa ville d'adoption, et de son pays. Il est plutôt dans le désenchantement, dans l'attente, que dans la critique. S'il souligne l'inachèvement des réformes, le maintien de l'apartheid social et spatial, la rareté du travail qui pousse à l'errance et à la délinquance loin de la famille et des lieux des racines et du contrôle social, il met aussi en avant, à travers les biographies de ses personnages, les progrès accomplis, la qualité du travail des jeunes policiers noirs qui manquent encore d'expérience mais ne sont pas corrompus, et qui ont la volonté de bien faire, d'apprendre. Certes, il est peu question dans ses romans de la vie dans les townships, de l'ampleur de l'épidémie de sida et de tuberculose, mais l'auteur évoque la volonté de s'élever par l'éducation, les valeurs rurales retrouvées (le jardin bien cultivé). Mais l'essentiel des intrigues se déroule dans des quartiers blancs, car les meurtriers, se recrutent, comme leurs victimes, parmi les Blancs. Thrillers, toujours, noirs, parfois, les romans de Deon Meyer nous entraînent dans les rues d'une ville violente, toute en contrastes, où Lamborghini et charrettes se côtoient, mais qui est aussi une ville solaire, qui aimante les forces vives du pays et attire les immigrants du monde entier.

\section{Les œuvres de Deon Meyer}

Feniks, 1996 ; Dead Before Dying, 1999 ; Jusqu'au dernier, 2002 (se passe en 1996)

Orion, 2000 ; Dead at Daybreak, 2000 ; Les soldats de l'aube, 2003 (se passe en 1999)

Proteus, 2003 ; Heart of the Hunter, 2003 ; L'âme du chasseur, 2005 (se passe en 2001)

Infata, 2005 ; Devil's Peak, 2007 ; Le pic du diable, 2007 (se passe en 2003)

Onsigbaar, 2007 ; Blood Safari, 2009 ; Lemmer l'invisible, 2008 (se passe en 2006-2007)

13 Uur, 2008 ; Thirteen Hours, 2010 ; 13 heures, 2010 (se passe en 2008-2009)

Spoor, 2010 ; Trackers, 2011 ; À la trace, 2012 (se passe en 2009-2010)

7 Dae, 2011 ; Seven Days, 2012 ; 7 jours, 2013 (se passe en 2010-2011).

\section{BIBLIOGRAPHIE}

Belaidi N., Berger M., Houssay-Holzschuch M., Ninot O., 2012. Cape Town. Périphéries postapartheid. In Chaléard, J.-L. (éd.), Les métropoles des Suds vues de leurs périphéries-Grafigéo, 34, p. 79-92.

Berger M., Houssay-Holzschuch M., Padovani F., 2014. Les évolutions récentes de la division sociale de l'espace dans deux villes globales du Sud, Shanghai et Le Cap. In Chaléard J.L. (dir.), Métropoles aux Suds, le défi des périphéries, Paris, Karthala, p. 63-80.

Houssay-Holzschuch M., 1966. Mythologies territoriales en Afrique du Sud, un essai de géographie culturelle. Paris, Presses du CNRS (Espaces et milieux), 120 p.

Houssay-Holzschuch M., 1999. Le Cap, ville sud-africaine. Ville blanche, vies noires. Paris, L'Harmattan, 276 p. 
Houssay-Holzschuch M., 2010. Crossing boundaries. t. 3 : Vivre ensemble dans l'Afrique du Sud postapartheid. HDR, Université Paris 1, 270 p.

Polar, le triomphe du mauvais genre, Le Monde Hors Série, 2014, 98 p.

Teppo A., Houssay-Holzschuch M., 2013. Gugulethu ${ }^{\mathrm{Tm}}$ : revolution for neoliberalism in a South African township. Canadian Journal of African Studies/La Revue canadienne des études africaines, 47:1, p. 51-74. http://dx.doi.org/10.1080/00083968.2013.770592

Thiébault C., Demets M., 2013. Polar. Panorama d'un genre majeur. Paris, La Martinière, 232 p.

\section{NOTES}

1. Cf. par exemple: Polar, le triomphe du mauvais genre, Le Monde Hors -Série, 2014.Ou encore : Thiébault C., Demets M., 2013. Polar. Panorama d'un genre majeur, La Martinière.

2. Auteur de Zulu (2008).

3. Cf. supra, Thiebault et Demets, préface ;

4. Deon Meyer et Mike Nicol.

5. Deon Meyer, Mike Nicol et Roger Smith sont les seuls à ce jour à avoir conquis un lectorat international.

6. 8 romans policiers publiés et traduits à ce jour, un neuvième (Cobra) à paraître en 2014 .

7. Nous avons pris le parti de citer les titres des romans dans leur traduction anglaise. Toutefois les références bibliographiques renvoient à la traduction française, et à sa publication en format de poche.

8. Je remercie tout particulièrement Myriam Houssay-Holzschuch, dont les travaux m'ont permis de décrypter l'univers social du Cap et nombre de références mémorielles dans les paysages urbains, et qui m'a patiemment pilotée dans l'exploration de la ville et la collecte photographique, y compris « dans ce genre de quartier, [où ] s'arrêter et regarder, ça ne se fait pas » (Trackers, p. 305). Et Olivier Ninot, pour les virées dans les endroits les plus improbables des délaissés urbains et des périphéries de l'Unicity.

9. Le site web de Deon Meyer (http://www.deonmeyer.com ) offre, pour les trois ouvrages cités, une sélection de photographies des lieux qui ont inspiré l'auteur.

10. Black Economic Empowerment.

11. Pour un aperçu de la géographie sociale du Cap, cf. par exemple Houssay-Holzschuch, 1999 et 2010 ; Belaidi et al., 2011 ; Berger, Houssay-Holzschuch, Padovani, 2013.

12. Les noms attribués aux voies, lors de la création des lotissements, font référence, par exemple, à des compositeurs italiens d'opéras, ou à des physiciens britanniques, ce qui ne manque pas d'étonner dans un tel environnement.

13. Reconstruction and Development Programme, qui comportait la construction massive de logements pour tenter de résorber la crise du logement, et de faire face à la multiplication des camps de squatters et des backyard shacks.

14. Dans un entretien avec une journaliste du Monde, le 19 novembre 2013, à l'occasion de l'Année de l'Afrique du Sud.

15. Tout comme Deon Meyer, qui a fait ses études dans cette ville et a été journaliste à Bloemfontein, avant de venir s'installer au Cap, où il réside à Melkbosstrand, sur la côte atlantique nord.

16. Entretien accordé à Babelio, http://www.babelio.com/auteur/Deon-Meyer/3019. Le site propose le texte intégral en version anglaise : Deon Meyer y détaille longuement les progrès accomplis depuis 1994.

17. South African Police Service. 


\section{RÉSUMÉS}

Parmi les auteurs sud-africains de romans policiers, Deon Meyer est celui dont les œuvres ont fait l'objet de la diffusion internationale la plus large. À travers huit romans, publiés entre 1996 et 2012 et comportant des personnages récurrents, il dresse un tableau de l'Afrique du Sud à une période charnière de son histoire. Ses intrigues, qui ont pour cadre principal la ville du Cap, conduisent le lecteur dans une grande diversité de quartiers et de milieux sociaux. Dressant un panorama des divisions spatiales, sociales et raciales dans l'Unicity, ses œuvres constituent un témoignage toujours précisément documenté sur la situation d'une métropole sud-africaine à l'heure de la globalisation.

Deon Meyer is the most famous among South African writers of detective stories. Eight novels with recurrent characters, published between 1996 and 2012, picture South Africa during a crucial period. The stories take place mainly in Capetown, in a wide range of communities. By picturing the social, spatial and racial divisions within the city, Deon Meyer depicts with high precision the state of a South African metropolis at the era of globalization.

\section{INDEX}

Mots-clés : roman policier, Afrique du Sud, Le Cap, géographie sociale post-apartheid, mondialisation

\section{AUTEUR}

\section{MARTINE BERGER}

Martine Berger, martine.berger@univ-paris1.fr, est Professeur des universités émérite et membre de l'UMR Prodig. Elle a publié récemment :

- Berger M., Aragau C., Rougé L., 2014. Vers une maturité des territoires périurbains ? Développement des mobilités de proximité et renforcement de l'ancrage dans l'ouest francilien, EchoGéo [En ligne], 27/2014, mis en ligne le 2 avril 2014. URL : http://echogeo.revues.org/13683

- Berger M., Houssay-Holzschuch M., Padovani F., 2014. Les évolutions récentes de la division sociale de l'espace dans deux villes globales du Sud, Shanghai et Le Cap. In Chaléard J.L. (dir.), Métropoles aux Suds, le défi des périphéries, Paris, Karthala, p. 63-80.

- Berger M., Rougé L. (dir.), 2011. Être logé, se loger, habiter, Regards de jeunes chercheurs, Paris, L'Harmattan, $320 \mathrm{p}$.

- Belaïdi N., Berger M., Houssay-Holzschuch M., Ninot O., 2011. Cape Town, périphéries postapartheid ». In Les métropoles des Suds vues de leurs périphéries, Grafigéo 2011-34, p. 79-92. 